\title{
BASIC AND TRANSLATIONAL-LIVER
}

\section{Oncogenic Mutations in Armadillo Repeats 5 and 6 of $\beta$-Catenin Reduce Binding to APC, Increasing Signaling and Transcription of Target Genes}

\author{
Pengyu Liu, ${ }^{1}$ Binyong Liang, ${ }^{2,3}$ Menggang Liu, ${ }^{1,4}$ Joyce H. G. Lebbink, ${ }^{5,6}$ Shan Li, ${ }^{1}$ \\ Manning Qian, ${ }^{2,7}$ Marla Lavrijsen, ${ }^{1}$ Maikel P. Peppelenbosch, ${ }^{1}$ Xin Chen, ${ }^{2}$ and Ron Smits ${ }^{1}$
}

${ }^{1}$ Department of Gastroenterology and Hepatology, Erasmus MC-University Medical Center, Rotterdam; ${ }^{2}$ Department of Bioengineering and Therapeutic Sciences and Liver Center, University of California, San Francisco, San Francisco, California; ${ }^{3}$ Hepatic Surgery Center, Department of Surgery, Tongji Hospital, Tongji Medical College, Huazhong University of Science and Technology, Wuhan, China; ${ }^{4}$ Department of Hepatobiliary Surgery, Daping Hospital, The Third Military Medical University, Chongqing, China; ${ }^{5}$ Department of Molecular Genetics, Cancer Genomics Netherlands, Erasmus MC, Rotterdam, The Netherlands; ' ${ }^{6}$ Department of Radiation Oncology, Erasmus MC, Rotterdam, The Netherlands; and ${ }^{7}$ The Clinical Medical Testing Laboratory, Northern Jiangsu People's Hospital and Clinical Medical College of Yangzhou University, Yangzhou, China
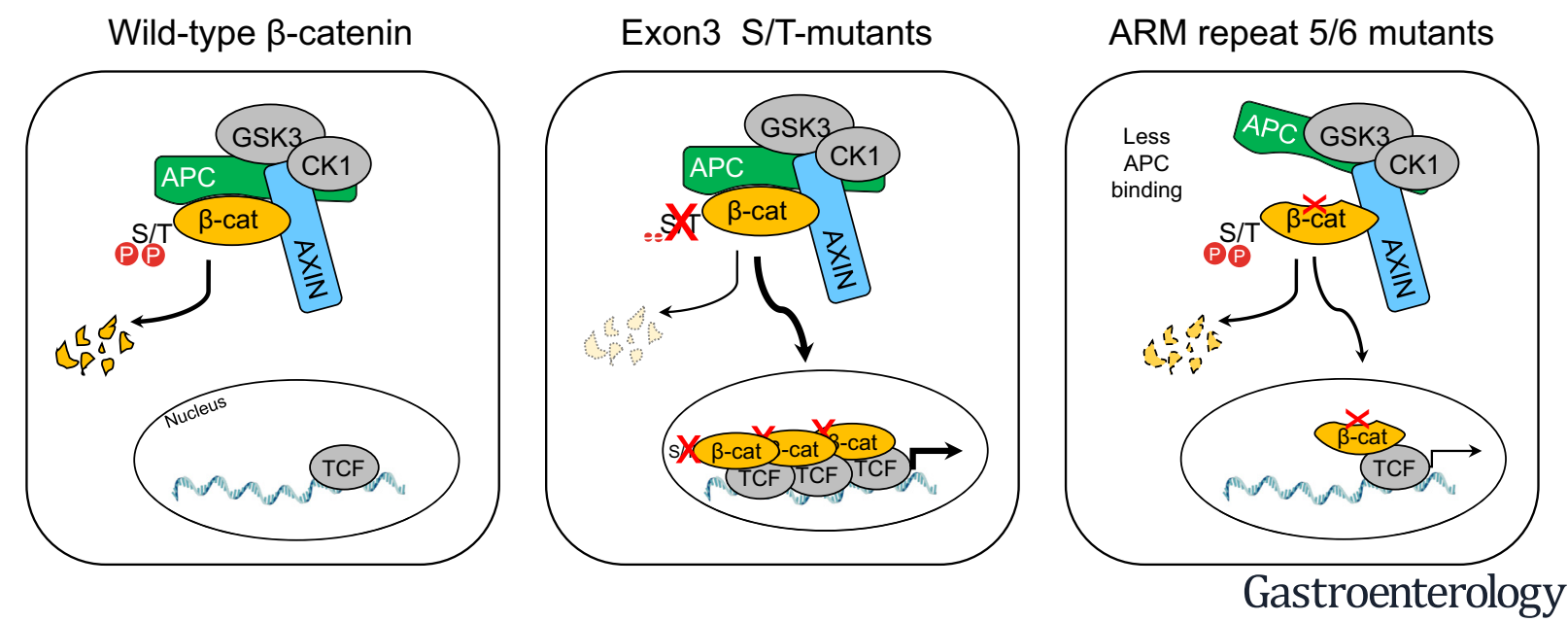

BACKGROUND \& AIMS: The $\beta$-catenin signaling pathway is one of the most commonly deregulated pathways in cancer cells. Amino acid substitutions within armadillo repeats 5 and 6 (K335, W383, and N387) of $\beta$-catenin are found in several tumor types, including liver tumors. We investigated the mechanisms by which these substitutions increase signaling and the effects on liver carcinogenesis in mice. METHODS: Plasmids encoding tagged full-length $\beta$-catenin (CTNNB1) or $\beta$-catenin with the K335I or N387K substitutions, along with MET, were injected into tails of FVB/N mice. Tumor growth was monitored, and livers were collected and analyzed by histology, immunohistochemistry, and quantitative reversetranscription polymerase chain reaction. Tagged full-length and mutant forms of $\beta$-catenin were expressed in HEK293, HCT116, and SNU449 cells, which were analyzed by immunoblots and immunoprecipitation. A panel of $\beta$-catenin variants and cell lines with knock-in mutations were analyzed for differences in N-terminal phosphorylation, half-life, and association with other proteins in the signaling pathway.
RESULTS: Mice injected with plasmids encoding K335I or N387K $\beta$-catenin and MET developed larger, more advanced tumors than mice injected with plasmids encoding WT $\beta$-catenin and MET. K335I and N387K $\beta$-catenin bound APC with lower affinity than WT $\beta$-catenin but still interacted with scaffold protein AXIN1 and in the nucleus with TCF7L2. This interaction resulted in increased transcription of genes regulated by $\beta$-catenin. Studies of protein structures supported the observed changes in relative binding affinities. CONCLUSION: Expression of $\beta$-catenin with mutations in armadillo repeats 5 and 6 , along with MET, promotes formation of liver tumors in mice. In contrast to N-terminal mutations in $\beta$-catenin that directly impair its phosphorylation by GSK3 or binding to BTRC, the K335I or N387K substitutions increase signaling via reduced binding to APC. However, these mutant forms of $\beta$-catenin still interact with the TCF family of transcription factors in the nucleus. These findings show how these amino acid substitutions increase $\beta$-catenin signaling in cancer cells. 
Keywords: Hepatocellular Carcinoma; Hepatocarcinogenesis; Gene Regulation; Mouse Model.

$\mathrm{T}$ he $\beta$-catenin signaling pathway is one of the most commonly deregulated pathways among cancers. ${ }^{1}$ In normal cells, $\beta$-catenin signaling is maintained at low levels by a destruction complex consisting of the adenomatous polyposis coli (APC) tumor suppressor, scaffold proteins axis inhibition protein (AXIN) 1 and 2, and the kinases glycogen synthase kinase 3 (GSK3) and casein kinase $1 \alpha$ (CK1 $\alpha$ ). In this complex, CK1 $\alpha$ initiates $\beta$-catenin (CTNNB1) phosphorylation at S45, followed by sequential GSK3 phosphorylation at T41, S37, and, finally, S33. Next, the phosphorylated 32DpSGXXpS37 motif promotes binding of the ubiquitin ligase $\beta$-transducin repeats-containing proteins ( $\beta$-TrCP [BTRC]), which targets $\beta$-catenin for proteasomal degradation. When cells are exposed to Wnt ligands, this $\beta$-catenin breakdown complex is temporarily inhibited, leading to the stabilization of $\beta$-catenin. As a result, it translocates into the nucleus and associates with members of the T-cell factor/lymphoid enhancer-binding factor (TCF/LEF) family of transcription factors, thus regulating the expression of specific downstream Wnt/ $\beta$-catenin target genes. ${ }^{2,3}$

In several tumor types, this pathway is constitutively activated through mutational (in)activation of one of the core elements of the destruction complex. In colorectal cancers, predominantly loss-of-function mutations are observed in the APC gene. For these mutations, it has become well accepted that they are selected on providing a just-right level of $\beta$-catenin signaling that is optimal for tumor initiation and progression. ${ }^{4}$ Other tumor types, such as hepatocellular and endometrial carcinomas, ${ }^{5-7}$ mainly acquire oncogenic $\beta$-catenin (CTNNB1) mutations at the $\mathrm{N}$-terminal $\mathrm{S} / \mathrm{T}$ phosphorylation residues, making the protein more resistant to proteolytic degradation. Recently, Rebouissou and coworkers ${ }^{8}$ showed that, for these CTNNB1 mutations, a clear genotype-phenotype correlation exists in liver cancer. In-frame exon 3 deletions that remove the entire $\mathrm{N}$-terminal phosphorylation domain and D32-S37 amino-acid alterations directly affecting the $\beta$-TrCP recognition motif lead to highly active $\beta$-catenin variants. T41 mutations were associated with moderate activity, whereas S45 mutations showed a weak but clear activation of the pathway. The reduced activity of the latter 2 mutations is most likely the result of residual phosphorylation of the $\beta$-TrCP interaction domain, leading to some breakdown of these mutants. ${ }^{9}$ This liver cancer study also included 2 more recently recognized mutational CTNNB1 hotspots, K335I and N387K, which are located in armadillo repeat domains 5 and 6 of the $\beta$-catenin protein, respectively. Currently, according to the Catalogue of Somatic Mutations in Cancer (COSMIC) Web site, ${ }^{10}$ mutations at these residues have been observed in more than 100 individual tumors, especially those of the liver, colon, and kidney (Figure 1 and Supplementary Table 1). They lead to a weak but significant enhancement of $\beta$-catenin signaling. ${ }^{8,11}$ However, the mechanism leading to their increased activity is still unknown. ${ }^{12}$ Here, by exploring

\section{WHAT YOU NEED TO KNOW \\ BACKGROUND AND CONTEXT}

The $\beta$-catenin signaling pathway is one of the most commonly deregulated pathways in cancer. Amino-acid alterations within armadillo repeats 5 and 6 are found in several tumor types, including liver tumors.

\section{NEW FINDINGS}

Expression of mutant forms of $\beta$-catenin found in several cancer types, along with MET, promoted formation of liver tumors in mice. The K335I or N387K substitutions increased signaling via reduced binding to APC, but these mutant forms of $\beta$-catenin still bound AXIN1 and the transcription factor TCF7L2 in the nucleus.

\section{LIMITATIONS}

These studies were performed in cell lines and mice.

IMPACT

These findings reveal how these amino acid substitutions in $\beta$-catenin increase signaling in cancer cells, including those of the liver in which they are most commonly observed.

potential mechanisms that may explain their increased signaling propensity, we identify a novel mechanism of enhanced $\beta$-catenin signaling.

\section{Methods}

\section{Catalogue of Somatic Mutations in Cancer Database Analysis}

To obtain the cancer-related CTNNB1 mutations depicted in Figure 1, we analyzed the COSMIC Web site (https://cancer. sanger.ac.uk/cosmic/gene/analysis?ln=CTNNB1), filtering it on amino acids 310-440. Data were updated until January 2019.

\section{Plasmids and Construction}

$\mathrm{N}$-terminal FLAG-tagged $\beta$-catenin variants were constructed by using the pcDNA $5^{\prime}$ UT-FLAG vector, as previously described. ${ }^{13}$ Briefly, the constructs of WT (WT), S33Y, and exon 3 deletion were generated by using the Gibson assembly method New England Biolabs (NEB). Based on the WT backbone, all other variants $(\mathrm{G} 34 \mathrm{~V}, \mathrm{~S} 37 \mathrm{~F}, \mathrm{~T} 41 \mathrm{~A}, \mathrm{~S} 45 \mathrm{P}, \mathrm{Y} 333 \mathrm{~F}$, E334K, K335I, K335T, R376H, W383G, W383R, N387K, and R582W) were constructed by using Q5 site-directed mutagenesis (NEB). The green fluorescent protein (GFP)-APC (11992167) plasmid was generated by cloning a 2.9-kilobase EcoRI

Abbreviations used in this paper: AAR, amino acid repeat; APC, adenomatous polyposis coli; AXIN1/2, axis inhibition protein 1/2; CK1 $\alpha$, casein kinase $1 \alpha$; COSMIC, Catalogue of Somatic Mutations in Cancer; CRISPR, clustered regularly interspaced short palindromic repeats; CTNNB1, $\beta$ catenin (cadherin-associated protein) $\beta 1$; GFP, green fluorescent protein; GSK3, glycogen synthase kinase 3; HCC, hepatocellular carcinoma; IP, immunoprecipitation; qRT-PCR, quantitative reverse-transcription polymerase chain reaction; TCF/LEF, T-cell factor/lymphoid enhancer-binding factor; $\beta$-TrCP, $\beta$-transducin repeats-containing protein; WT, wild type.

\section{Most current article}

\section{2020 by the AGA Institute} $0016-5085 / \$ 36.00$

https://doi.org/10.1053/j.gastro.2019.11.302 


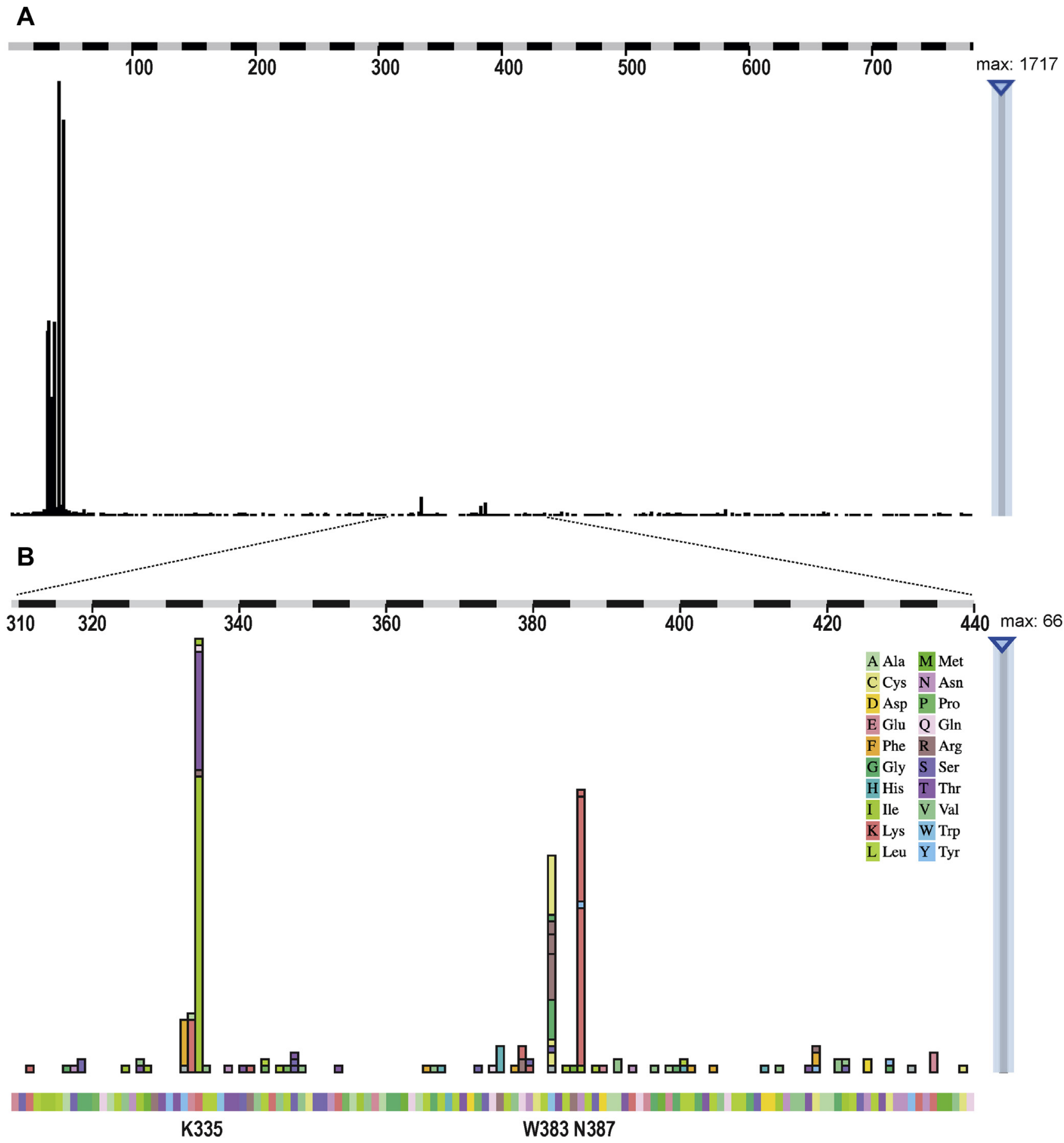

Figure 1. Oncogenic hotspot mutations observed in armadillo repeats 5 and 6 of $\beta$-catenin. Three amino acids are commonly mutated in cancers: K335, W383, and N387. (A) An overview of all reported amino acid substitutions along the $\beta$-catenin protein. $(B)$ Mutations restricted to amino acids 310-440. Details such as involved cancer types can be explored interactively on the COSMIC Web site, but are also presented in Supplementary Table 1. The following alterations have been observed at least 4 times: Y333F, 7 times; E334K, 8 times; K335T, 18 times; K335I, 46 times; R376H, 4 times; W383C, 12 times; W383R, 12 times; W383G, 7 times; and N387K, 40 times.

fragment of mouse $A p c$ into pEGFP-C1. It encodes all 20 amino acid $\beta$-catenin-binding repeats and all AXIN-binding domains of APC. pcDNA3.1 myc-m $\beta$ TrCP WD1-7 (241-569) was a gift from Michael Ruppert (\#62977; Addgene, Watertown, MA). ${ }^{14}$ pCS2MT mouse Axin (Axin MTFu1) was a gift from Frank
Costantini (Addgene \#21287) and encodes an N-terminal 6xMyc-tagged mouse AXIN1 variant of amino acid $832 .{ }^{15}$ The pT3-EF1 $\alpha$ - $\beta$-catenin-K335I and pT3-EF1 $\alpha$ - $\beta$-catenin-N387K constructs were generated by subcloning a Bsu36I-EcorI fragment in the pT3-EF1 $\alpha$ - $\beta$-catenin-WT plasmid (with N-terminal 
Myc-tag). pT3-EF1 $\alpha$-c-Met and pCMV/sleeping beauty transposase (pCMV/SB) plasmids have been described previously. ${ }^{16}$

\section{Mice and Hydrodynamic Tail Injection}

Hydrodynamic tail vein injection was performed basically as previously described. ${ }^{17-19}$ Briefly, $20 \mu \mathrm{g}$ of either pT3-EF1 $\alpha$ $\beta$-catenin-WT, K335I, or N387K construct was mixed with 20 $\mu \mathrm{g}$ pT3- EF1 $\alpha$-c-Met and $1.6 \mu \mathrm{g}$ pCMV/SB in $2 \mathrm{~mL}$ saline. This solution was injected in the lateral tail vein of 6- to 8-week-old WT FVB/N mice purchased from The Jackson Laboratory (Sacramento, CA). Mice were monitored via abdominal palpation every 3 days. The mice were killed and were considered to be dead when they developed a palpable abdominal mass, based on the Institutional Animal Care and Use Committee protocol. Mice were housed and fed in accordance with protocols approved by the Committee for Animal Research at the University of California, San Francisco. Livers and tumors were analyzed basically as previously described (see Supplementary Methods). ${ }^{17-19}$

\section{Clustered Regularly Interspaced Short Palindromic Repeats/Cas9 Mediated Knock-In of CTNNB1 Mutations}

Previously, we generated SNU449 clones in which the AXIN1 p.R712* mutation was successfully repaired with clustered regularly interspaced short palindromic repeats (CRISPR)/Cas9 technology (manuscript submitted). Clone-20 thereof and HEK293 cells were used to knock in $\beta$-catenin S37F and K335I mutations, as described in the Supplementary Methods.

\section{Half-Life Determination}

$\mathrm{N}$-terminal FLAG-tagged $\beta$-catenin variant plasmids (200 ng) were cotransfected into HEK293 cells with pEGFP-C1 (50 ng) using FuGENE HD. The pEGFP-C1 plasmid generates a highly stable GFP protein serving as loading control. After 24 hours, the cells were treated with cycloheximide $(50 \mu \mathrm{g} / \mathrm{mL})$ for $0,30,60,90,120$, and 150 minutes. Next, cells were washed with cold phosphate-buffered saline 2 times and lysed in $2 \times$ Laemmli sample buffer (120 mmol/L Tris-Cl pH 6.8, 20\% glycerol, $4 \%$ sodium dodecyl sulfate) with $0.1 \mathrm{~mol} / \mathrm{L}$ dithiothreitol, and heated for 5 minutes at $95^{\circ} \mathrm{C}$.

\section{Immunoprecipitation}

For the immunoprecipitation (IP) assays using expression plasmids, HEK293 or HCT116 cells were seeded in 6-well plates before transfection. When reaching $60 \%-80 \%$ confluence, $1 \mu \mathrm{g} \mathrm{N}$-terminal FLAG-tagged $\beta$-catenin variant plasmids were cotransfected with either $1 \mu \mathrm{g}$ of APC-, AXIN- or $\beta$ TrCPexpression plasmids using FuGENE HD (Promega, The Netherlands). After 24 hours, FLAG-tagged $\beta$-catenin was immunoprecipitated with ANTI-FLAG M2 Affinity Gel (SigmaAldrich, St Louis, MO). Knock-in cell lines were seeded in 15$\mathrm{cm}$ round plates, lysed, and incubated with primary antibody, which was immunoprecipitated with Pierce Protein A/G Magnetic beads (Thermo Fisher Scientific, Waltham, MA). Details of the IP protocols can be found in the Supplementary Methods.

\section{Western Blotting, Antibodies, and Quantification}

Fluorescent Western blotting and quantitative analysis were performed basically as previously described. ${ }^{20}$ Primary antibodies are listed in the Supplementary Methods. As secondary antibodies, we used anti-rabbit or anti-mouse IRDyeconjugated antibodies (LI-COR Biosciences, Lincoln, NE). Protein intensity was detected with the Odyssey 3.0 Infrared Imaging System and analyzed by Image Studio Lite, version 5.2 (LI-COR). For APC Western blot analysis, we used Immobilon ECL Ultra HRP substrate (Merck Millipore, Billerica, MA). Membranes for ECL detection were blocked and incubated using Immobilon Block-PO reagent (Merck Millipore).

\section{$\beta$-Catenin Reporter Assays}

HEK293 cells were seeded in 24-well plates to reach approximately $50 \%-70 \%$ confluency on the day of transfection. Each well was transfected with $5 \mathrm{ng}$ of the $\beta$-catenin expression vectors, 250 ng TOPflash or FOPflash, and 10 ng CMV-Renilla using Fugene HD (Promega, Madison, WI). For the knock-in HEK293 and SNU449 cells, we used the more sensitive Wnt-Responsive Element/ Mutant Responsive Element (WRE/MRE) reporters, using 12-well and 6-well plates, respectively. After 2 days, luciferase activities were measured in a LumiStar Optima luminescence counter (BMG LabTech, Offenburg, Germany) and normalized for transfection efficiency by using the Dual Luciferase Reporter Assay system (Promega), according to the manufacturer's instruction. Transfections were performed in triplicate, and the mean and standard error were calculated for each condition. The $\beta$-catenin reporter activities are shown as TOP/FOPflash or WRE/MRE ratios.

\section{Structure Analysis}

Structures were analyzed and figures were created with the PyMOL Molecular Graphics System, version 2.0 (Schrödinger, New York, NY). Overlays were created by superimposing $\beta$-catenin (chain A) from the $\beta$-catenin-TCF complex (1G3J.pdb), $\beta$-catenin-E-cadherin complex (1I7W.pdb), or the $\beta$-cateninAXIN complex (1Q27) onto chain A of the $\beta$-cateninphosphorylated 20-amino acid APC complex (1TH1.pdb).

\section{Statistical Method}

Expression differences tested by quantitative reversetranscription polymerase chain reaction (qRT-PCR), and luciferase assays were tested by unpaired $t$ test. Statistical differences are depicted as follows: ${ }^{*} P<.05$; ${ }^{* *} P<.01$; ${ }^{* * *} P<.001$; ${ }^{* * * *} P<.0001$. All statistical analyses were performed using GraphPad Prism 6 (GraphPad Software, San Diego, CA).

\section{Results}

\section{K335I and N387K $\beta$-Catenin Variants Are Potent Inducers of Hepatocellular Carcinoma Formation in Mice}

To directly demonstrate the oncogenic potential of the $\beta$-catenin K335I and N387K variants, we hydrodynamically injected them in the tail vein of mice. ${ }^{21}$ WT $\beta$-catenin was taken along as control. All variants were cotransfected with c-Met because previous work showed that oncogenic $\beta$-catenin alone is insufficient for hepatocellular carcinoma (HCC) development. ${ }^{16,17}$ In strong contrast to the mice injected with 
WT $\beta$-catenin, all K335I/N387K injected mice became moribund 6-7 weeks after injection, showing a massive increase in liver weight and tumor burden (Figure $2 A$ ). Histologically, we observed the presence of well- to moderately differentiated HCC with solid or macrotrabecular growth patterns (Figure $2 A$ and Supplementary Figure 1), in accordance with previous observations. ${ }^{16,17}$ Lesions are positive for the hepatocyte marker HNF4 $\alpha$ but negative for the biliary marker CK19 (Supplementary Figure 2). In WT injected mice, only occasional HCC-like foci were observed. This marked difference in oncogenic potential was confirmed by immunohistochemical analysis of the proliferation marker Ki-67 (Figure $2 B$ and $C$ ) and qRT-PCR analysis showing an elevated expression of the HCC markers Gpc3 and Afp (Figure 2D).

Staining for $\beta$-catenin showed a predominant membranous location (Figure $3 A$ ). Occasionally a weak cytoplasmic or nuclear staining was observed, especially for the $\mathrm{N} 387 \mathrm{~K}$ variant. This result was confirmed with an antibody detecting the Myc tag present in the construct (Figure $3 A$ ). The lack of a prominent nuclear accumulation is in line with previous observations for the weak $\beta$-catenin S45Y variant. ${ }^{17}$ Nevertheless, we observed a strong increase in the (liver-specific) $\beta$-catenin target genes Axin2, Tbx3, and Glul (Figure $3 B$ ), the latter also confirmed by immunohistochemical staining for glutamine synthetase (Figure $3 C$ ).

Taken together, these results show that both the K335I and $\mathrm{N} 387 \mathrm{~K}$ variants, compared with WT $\beta$-catenin, have a strong potential to induce liver tumor formation in mice, associated with a clear induction of target gene expression.

\section{Half-Life of $\beta$-Catenin Mutants Correlates With Their Activation Potential}

The signaling activity of $\beta$-catenin is strongly regulated by proteolytic degradation. Therefore, we analyzed the half-lives of various commonly observed $\beta$-catenin variants in comparison with WT protein (Supplementary Figure 3). Interestingly, K335I and $\mathrm{N} 387 \mathrm{~K}$ variants show a similar protein stability as WT $\beta$-catenin, with half-lives between 65 and 100 minutes. The S45P mutation increases the half-life up to 110 minutes, and mutations affecting the D32-S37 $\beta$-TrCP binding motif and the exon 3-deletion variant extends it to more than 2 hours. Thus, these analyses show that protein stability largely correlates with the reported signaling activities; that is, mutants with weak enhanced signaling capabilities show a turnover comparable to the WT protein, whereas more active signaling variants show a clearly increased half-life.

\section{K335 and N387 Variants Are Not Affected in $N$-Terminal Phosphorylation and $\beta$-TrCP Binding}

$\beta$-catenin protein stability is largely regulated by sequential $\mathrm{N}$-terminal $\mathrm{S} / \mathrm{T}$ phosphorylation. Hence, we determined the phosphorylation status of $\beta$-catenin variants transfected in HEK293 cells (Figure 4A). The exon 3-deletion variant lacking all $\mathrm{S} / \mathrm{T}$ residues cannot be recognized by any of the phospho-antibodies. All other variants are readily phosphorylated at S45 comparable to the WT protein, except for the S45P mutant. In contrast, the GSK3-mediated phosphorylation at residues $\mathrm{T} 41, \mathrm{~S} 37$, and S33 is reduced in all
S33 to S45 mutants. In accordance with previous reports, we still observed some residual S/T phosphorylation in all these variants. ${ }^{9}$ Importantly, in this overexpression assay, the K335 and N387 mutants showed no obvious deviation from the phosphorylation pattern observed for the WT protein.

Next, we determined the binding capacity of $\beta$-TrCP to each variant in HEK293 cells. Our IP protocol resulted in a weak unspecific binding of Myc-tagged $\beta$-TrCP in the negative control (lane 2 of Figure $4 B$ ). For the S33-S45 mutants, similar or, at most, slightly increased levels of coimmunoprecipitated $\beta$-TrCP were observed, whereas both K335 and N387 mutants showed equal binding capabilities, comparable to the WT protein. Overall, these analyses show that K335I and $\mathrm{N} 387 \mathrm{~K}$ mutant proteins are not seriously affected in $\mathrm{N}$ terminal phosphorylation and binding to $\beta$-TrCP.

\section{K335 and N387 Variants Show Reduced Binding to the Adenomatous Polyposis Coli Protein}

Because protein half-life, N-terminal phosphorylation, and $\beta$-TrCP binding are not clearly affected for the K335I and $\mathrm{N} 387 \mathrm{~K}$ variants, we sought for an alternative explanation explaining their increased signaling behavior. Both residues are located in armadillo repeats 5 and 6 of $\beta$-catenin in the center of the protein. In total, 12 armadillo repeats (residues 141-664) form a superhelix with a positively charged groove, which can associate with a large number of proteins. ${ }^{2}$ We focused on wellestablished binding partners at the cell membrane, nucleus, and within the destruction complex, that is, E-cadherin, TCF7L2 (also known as TCF4), AXIN1, and APC. Except for AXIN1, these have all been shown to bind an extended area encompassing both K335 and N387 residues (Figure 5A). Within epithelial cells, most $\beta$-catenin is captured at the cell membrane through interactions with cadherins. Hence, a reduced binding affinity between $\beta$-catenin and E-cadherin is expected to increase the signaling pool of $\beta$-catenin, possibly leading to enhanced nuclear signaling. However, IP of WT and the K335I and N387K variants from HCT116 cells showed no altered binding to Ecadherin (Figure $5 B$ ). Alterations in binding to TCF7L2 within the nucleus are also expected to affect activation of $\beta$-catenin target genes, but again, we failed to show any difference between the tested variants (Figure $5 B$ ). Likewise, binding of cotransfected MYC-tagged AXIN1 was not affected by both mutations (Figure $5 C$ ). In contrast, binding of a cotransfected GFP-tagged APC fragment was strongly impaired for the K335I and N387K mutants (Figure 5D). Taken together, these IP experiments show that, compared with WT $\beta$-catenin, both mutants associate less strongly with APC, one of the core proteins of the destruction complex.

\section{Endogenous Expression in Cell Lines Confirms Increased Signaling and Reduced Affinity for Adenomatous Polyposis Coli Protein}

Because overexpression may affect the stoichiometry of the $\beta$-catenin breakdown complex, we wished to confirm our results at endogenous expression levels. Previously, we generated SNU449 liver cancer cells in which the AXIN1 p.R712* mutation was successfully repaired with CRISPR/ Cas9 technology (manuscript submitted), so that no known 


\section{A $\beta$-catenin-WT/c-Met}
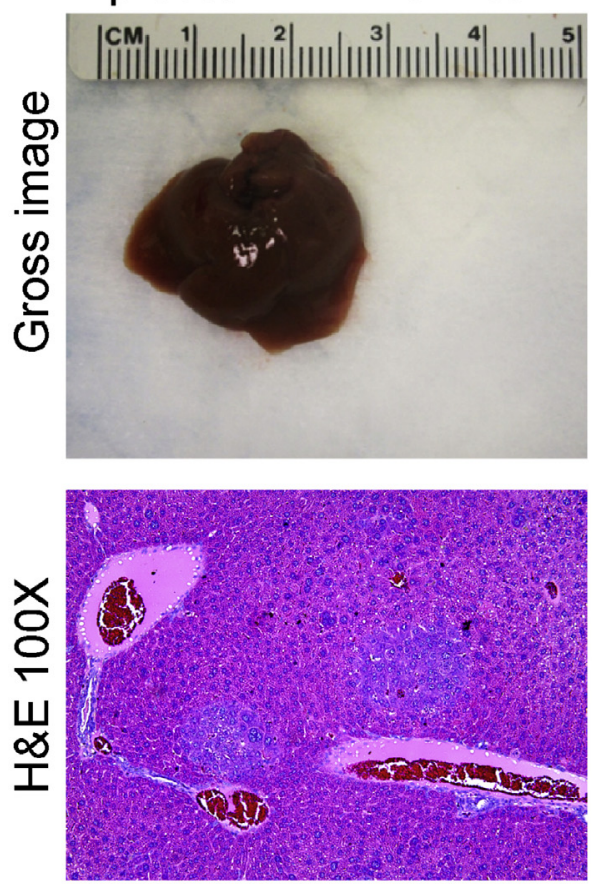

B $\beta$-catenin-WT/c-Met

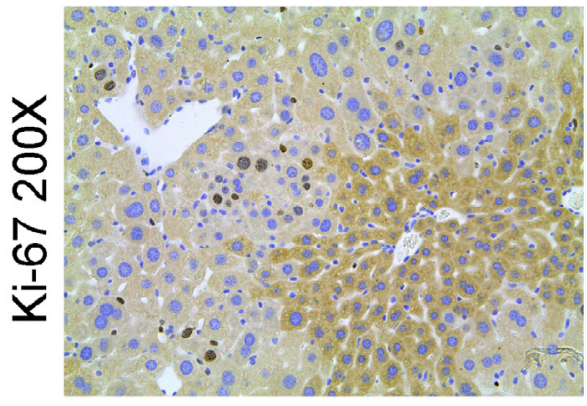

C

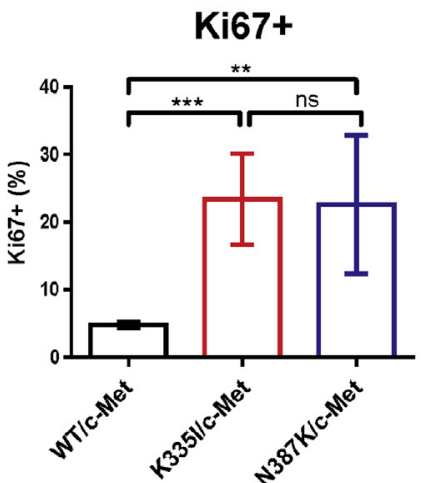

ß-catenin-K335I/c-Met

| ||||||||||||||||||||||||||||||||||||||||||||||||||||
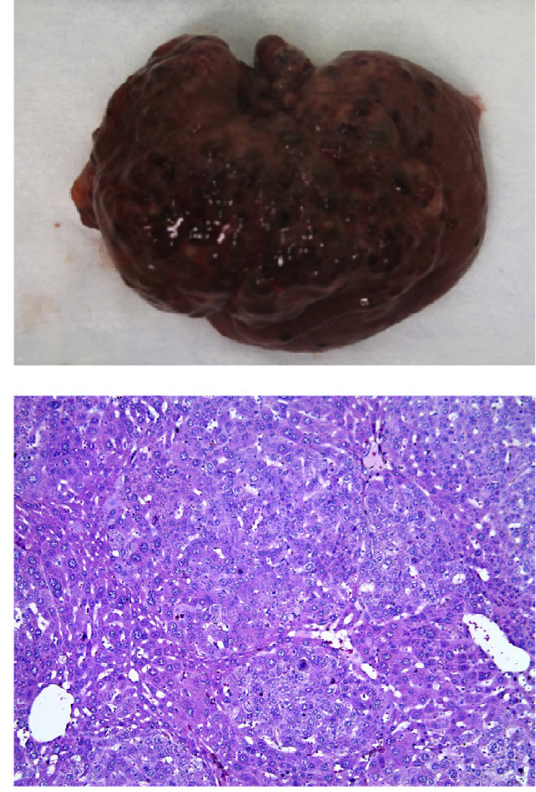

$\beta$-catenin-K335I/c-Met

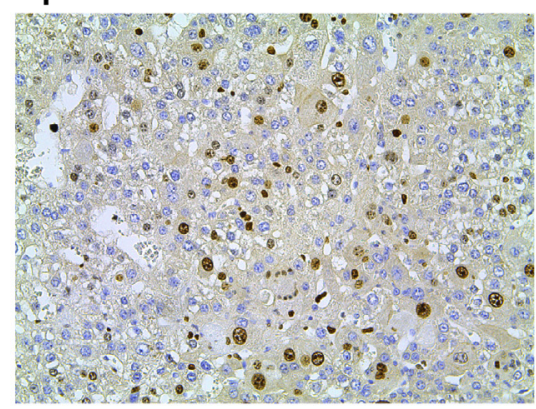

D

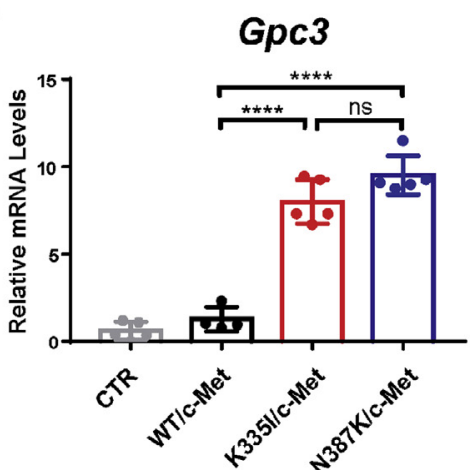

ß-catenin-N387K/c-Met
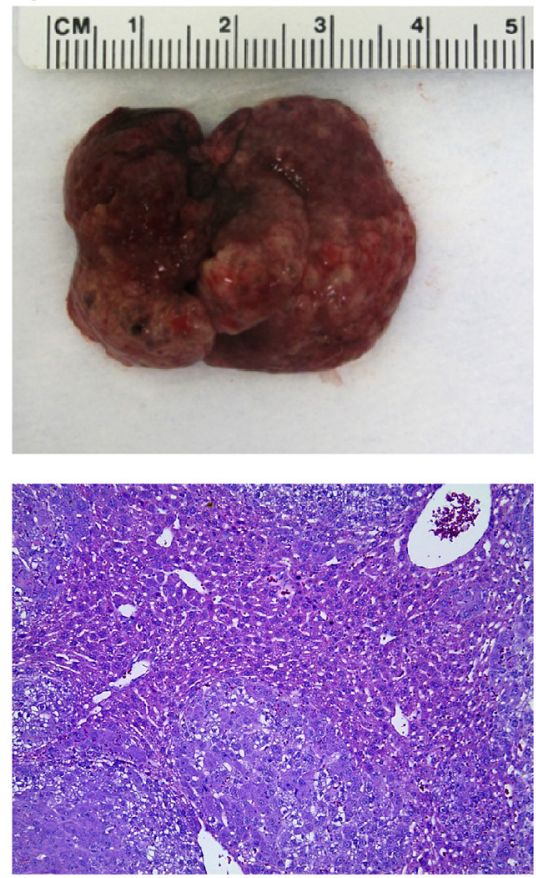

$\beta$-catenin-N387K/C-Met
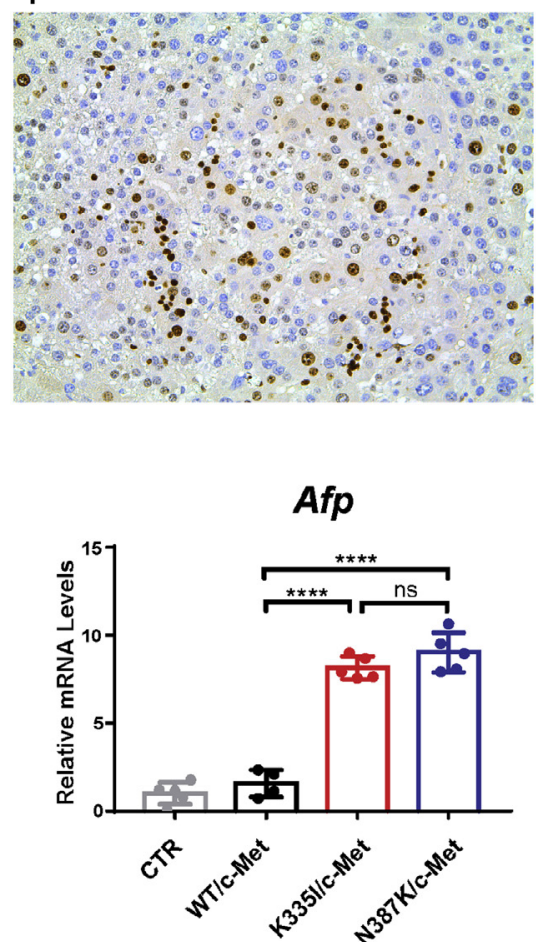

Figure 2. K335I and N387K $\beta$-catenin variants are potent inducers of $\mathrm{HCC}$ development in mice. $(A)$ Hydrodynamic tail vein injection of $\beta$-catenin-K335I and N387K variants resulted in a strong increase of liver weights within 6-7 weeks, in contrast to WT $\beta$-catenin-injected animals ( $\mathrm{n}=5$ each). All variants were cotransfected with $\mathrm{c}-\mathrm{Met}$. H\&E staining showed multiple large foci of well-differentiated HCCs with solid or macrotrabecular growth patterns. (B) Immunohistochemical analysis for the proliferation marker Ki67 and (C) quantification of 5 representative fields. (D) Expression of HCC markers Gpc3 and Afp was evaluated by qRT-PCR in normal (CTR) and transfected livers. All data are presented as mean \pm standard deviation. ${ }^{\star} P<.05$; ${ }^{* \star} P<.01 ;{ }^{* \star \star} P<.001 ;{ }^{* \star \star} P<.0001 ;$ ns, nonsignificant. 
$\beta$-catenin activating mutations remain. In these and HEK293 cells, we successfully knocked in the K335I mutation. For direct comparison, we also generated cells carrying the strong S37F mutation (see Supplementary Table 2 for clone details).
Attempts to generate $\mathrm{N} 387 \mathrm{~K}$ knock-in cell lines failed. Western blot analysis of SNU449 mutant cells showed a modest increase in total $\beta$-catenin levels, especially for the S37F mutant cells (Figure 6A), whereas in HEK293, this was
A $\beta$-catenin-WT/c-Met
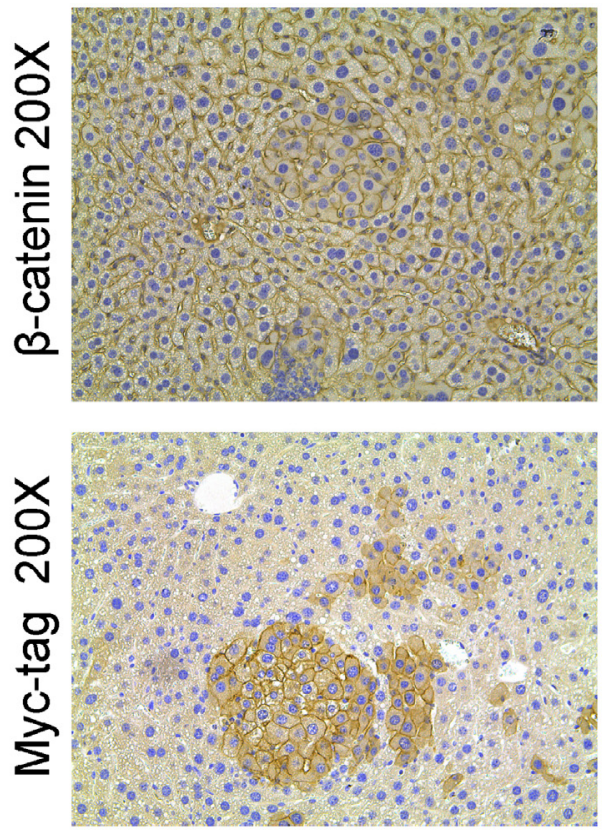

B

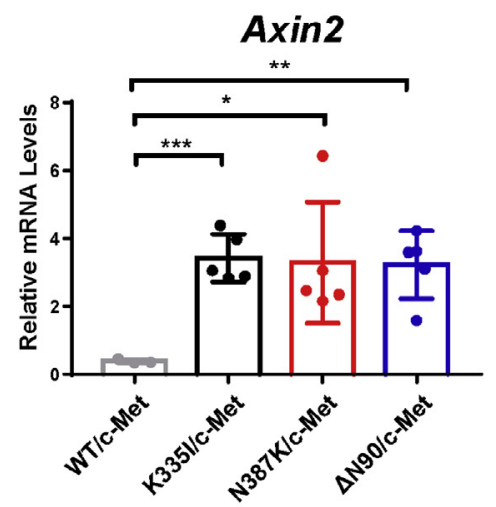

C $\beta$-catenin-WT/c-Met

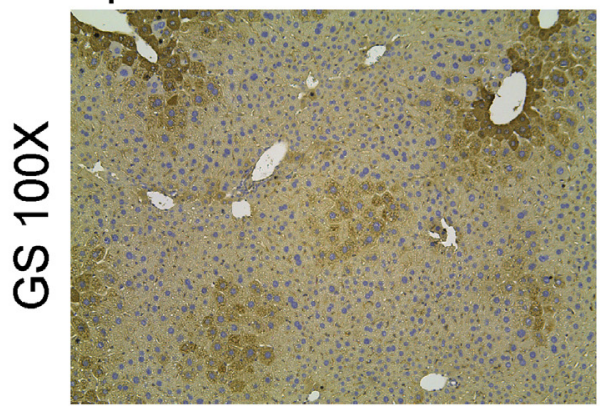

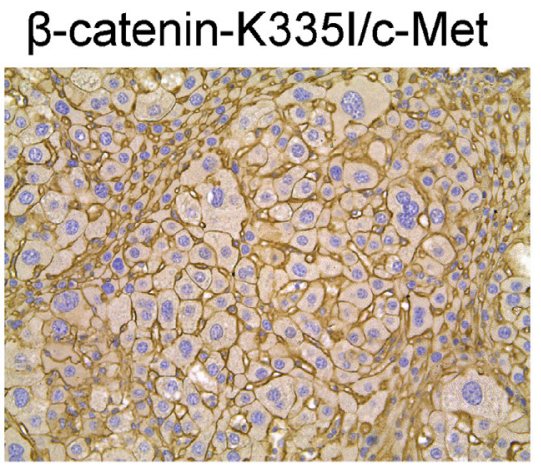
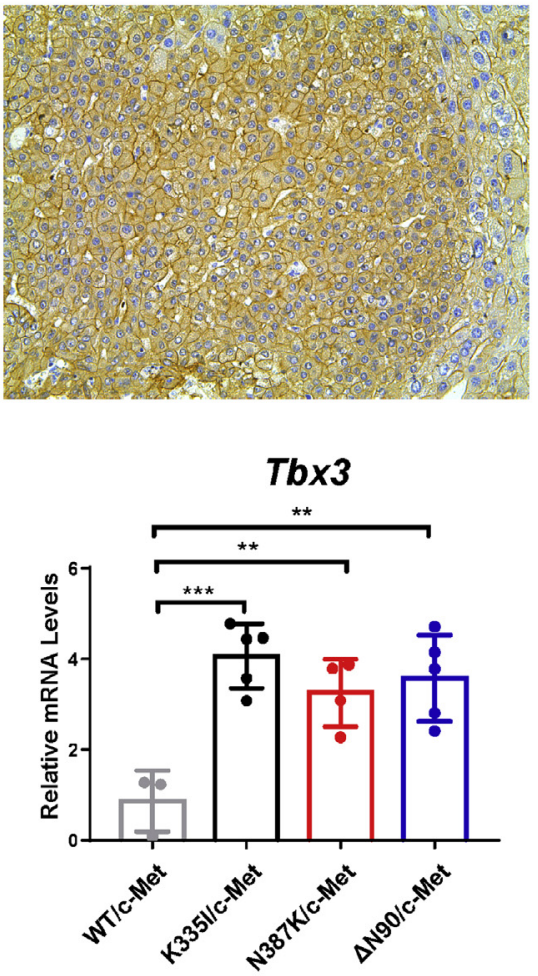

ß-catenin-K335I/c-Met

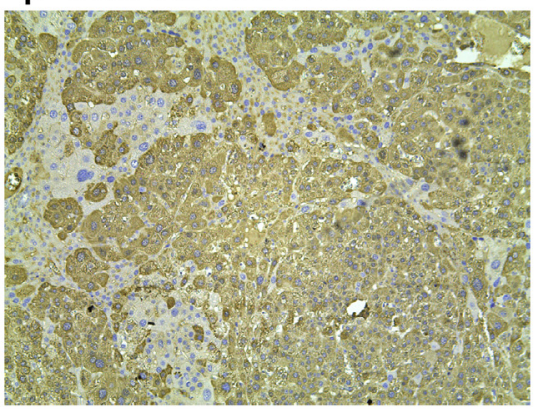

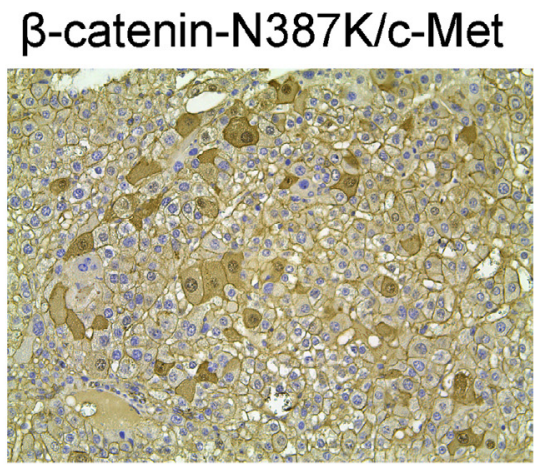
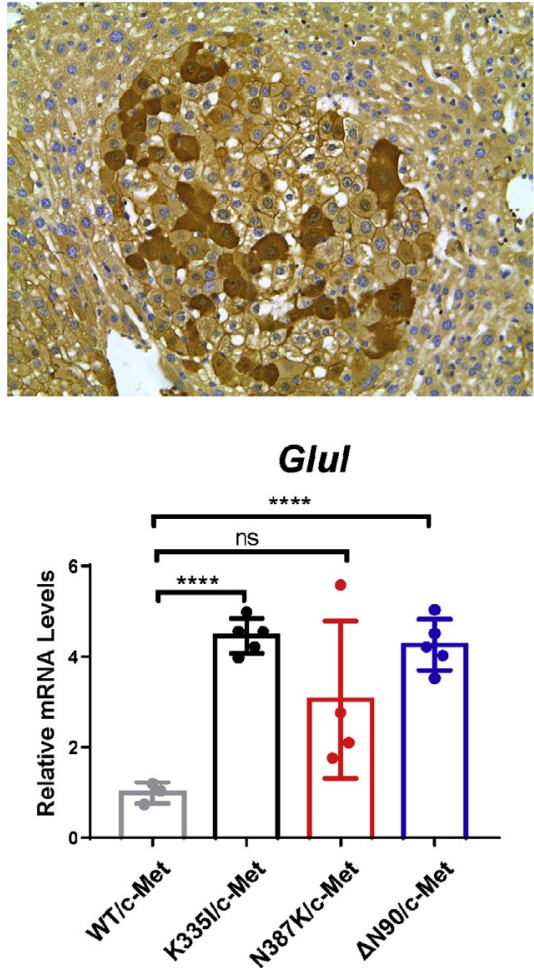

$\beta$-catenin-N387K/c-Met

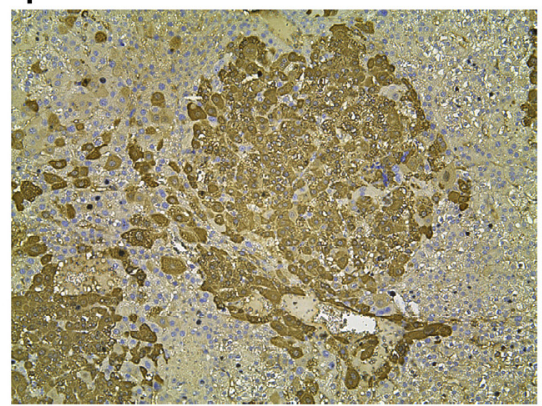

Figure 3. Immunohistochemical analysis of $\beta$-catenin patterns and target gene expression. $(A) \mathrm{IHC}$ for $\beta$-catenin and associated Myc-tag shows a predominant membranous location for transfected WT, K335I, and N387K variants. Occasionally, a stronger cytoplasmic staining is observed, especially for the N387K variant. (B) qRT-PCR analysis of $\beta$-catenin target genes Axin2, Tbx3, and Glul. RNA isolated from previously injected $\beta$-catenin- $\Delta 90 \mathrm{~N} / \mathrm{c}$-Met animals was used as positive control. ${ }^{17}$ (C) Immunohistochemical confirmation of glutamine synthetase (GS) expression. 


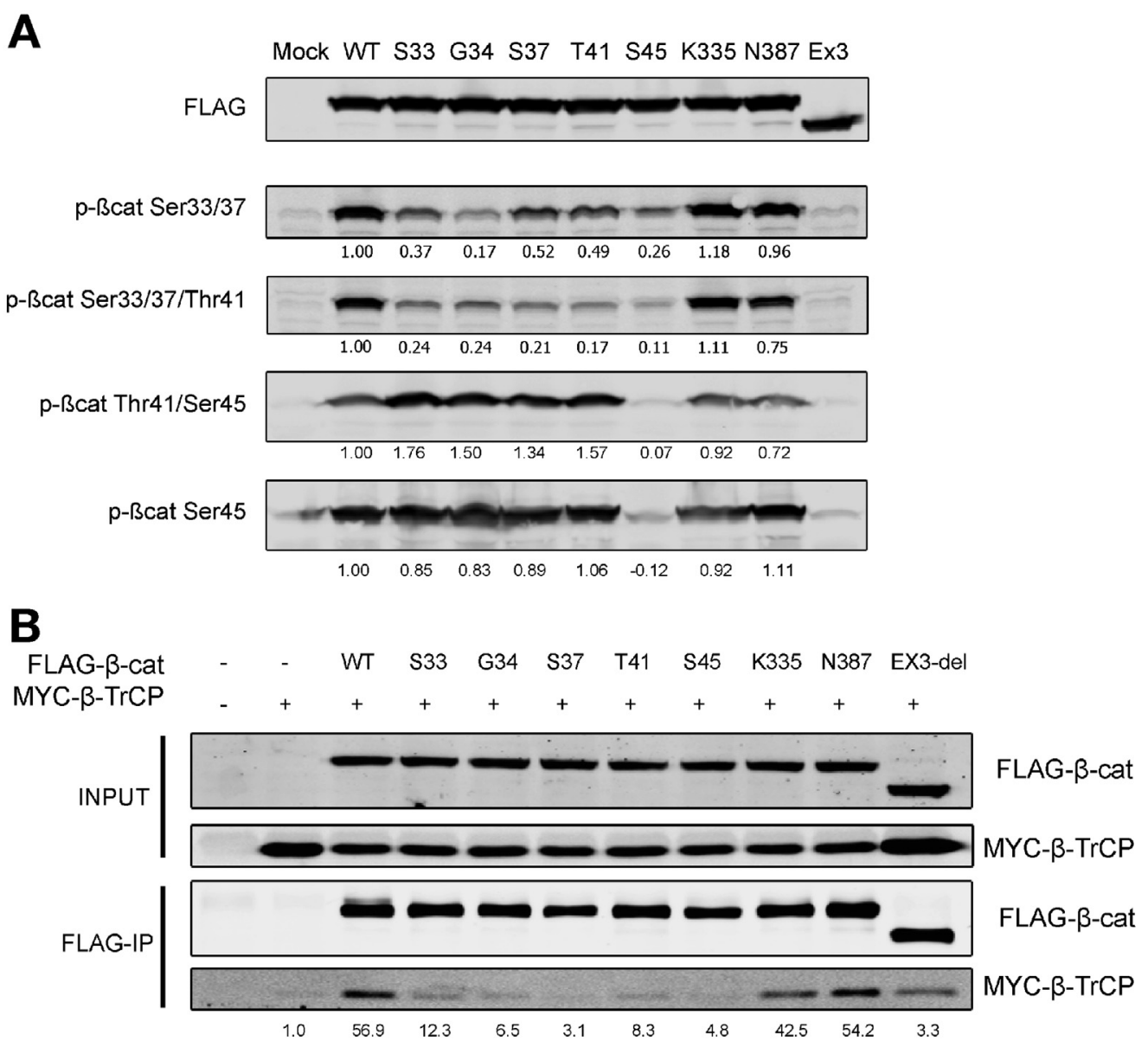

Figure 4. K335 and N387 variants are not affected in N-terminal phosphorylation and $\beta$-TrCP binding. (A) FLAG-tagged $\beta$-catenin variants were transfected in HEK293 and subjected to Western blotting with phospho-specific $\beta$-catenin antibodies (Supplementary Table 7). Endogenous $\beta$-catenin is visible in the mock and exon 3-deletion transfected lanes, which was subtracted from the transfected variants. Signal of WT protein was set to 1, to which all other values were normalized. K335I and N387K variants are similarly phosphorylated as WT protein. (B) FLAG-tagged $\beta$-catenin variants were cotransfected with Myctagged $\beta$-TrCP into HEK293 cells. Two hours before FLAG-IP, cells were treated with the proteasome inhibitor MG132. The amount of coimmunoprecipitated $\beta$-TrCP was determined after normalization with $\beta$-TrCP input signal. Signal of IP obtained with Myc-tagged $\beta$-TrCP alone was set to 1 . K335I and N387K variants are not affected in their association with $\beta$-TrCP.

the case only for S37F clones. All K335I clones showed a significant increase in $\beta$-catenin reporter activity (Figure 6B), although not as pronounced when compared with the S37F variant. These results were confirmed with AXIN2 qRT-PCR (Supplementary Figure 4). IP of endogenous APC using 2 independent antibodies confirmed the reduced binding of the K335I variant (Figure 6C). These results confirm the increased signaling propensity of the armadillo variants through reduced APC binding at endogenous levels as well.

\section{Protein Structures of $\beta$-Catenin Complexes Provide a Rationale for Reduced Binding of Mutant $\beta$-Catenin Selectively to the Adenomatous Polyposis Coli Protein}

To identify potential mechanisms underlying the differential binding of AXIN1, APC, TCF7L2, and E-cadherin to both mutants, we analyzed available protein structures of $\beta$-catenin in complex with fragments of these proteins (Supplementary Figure $5 A$ ) ${ }^{22-25}$ In all complexes,
$\beta$-catenin-K355 forms a hydrogen bond with a backbone carbonyl oxygen of the bound partner protein (Supplementary Figure 5B-D). In addition, K335 forms specific interactions with sidechains from the bound proteins, which are different for each partner and depend on its phosphorylation status. The 7 20-amino acid repeats (AARs) of APC contain a conserved SxxxSLSSL motif that is phosphorylated on multiple serine residues by GSK3/CK1 when APC is associated with AXIN, which strongly increases the binding to $\beta$-catenin. ${ }^{25-27}$ Interestingly, in the $\beta$-cateninAPC complex, the positively charged K335 sidechain forms ionic interactions and hydrogen bonds with 2 negatively charged phosphorylated serines (pS1504 and pS1507) in the APC 20-AAR motif (Supplementary Figure 5B), all of which are lost when mutated to isoleucine or threonine. In the $\beta$-catenin-TCF complex, no serines are available within this region for phosphorylation; instead, K335 forms a single ionic interaction with the negatively charged sidechain of D40 (Supplementary Figure 5C). E-cadherin carries a phosphorylated serine (S692) at the corresponding position 
A

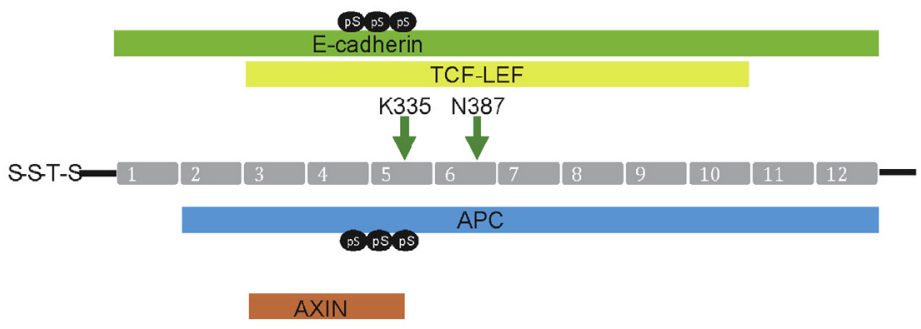

B

\begin{tabular}{|c|c|c|c|}
\hline FLAG-ßcat-WT & + & - & - \\
\hline FLAG-ßcat-335 & - & + & - \\
\hline FLAG-ßcat-387 & - & - & + \\
\hline MYC-AXIN1 & - & - & - \\
\hline
\end{tabular}

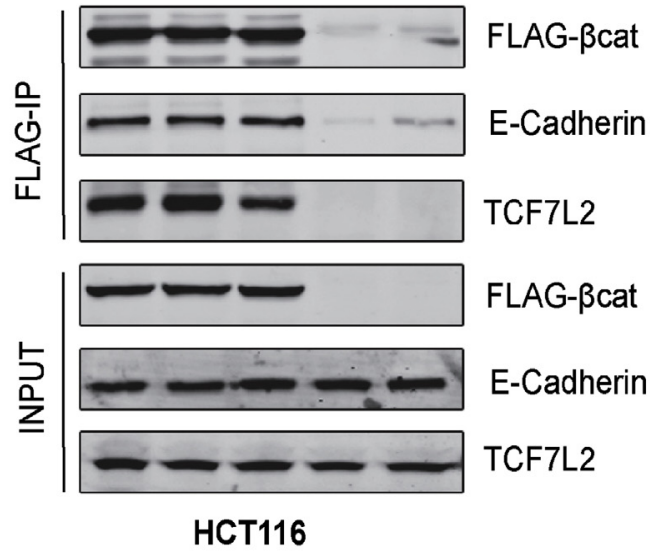

D

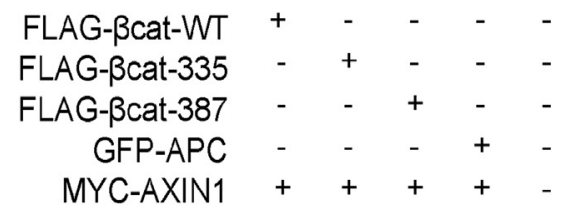

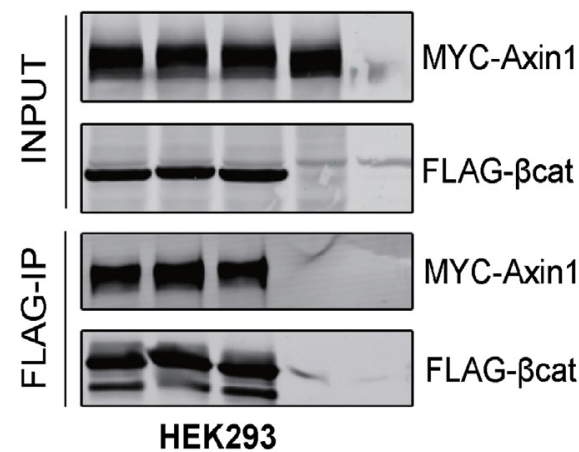

$\begin{array}{rlllll}\text { FLAG- } \beta \text { cat-WT } & + & - & - & - & - \\ \text { FLAG- } \beta \text { cat-335 } & - & + & - & - & - \\ \text { FLAG- } \beta \text { cat-387 } & - & - & + & - & - \\ \text { GFP-APC } & + & + & + & + & - \\ \text { MYC-AXIN1 } & - & - & - & + & -\end{array}$
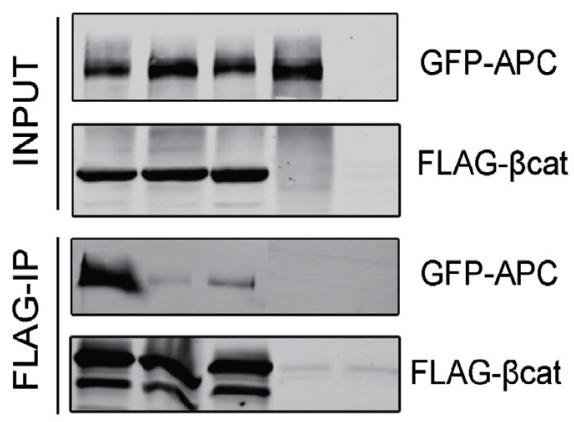

HEK293

Figure 5. K335I and N387K variants selectively show reduced binding to APC. $(A)$ Schematic representation of the reported binding domains of APC, AXIN, TCF/LEF, and E-cadherin to the armadillo repeat region of $\beta$-catenin. Positions of K335I and N387K mutations are indicated by arrows. pS indicates serine phosphorylation present at binding interface of APC or E-cadherin. (B) FLAG-tagged WT, K335I, and N387K $\beta$-catenin variants were transfected in HCT116 cells. Myc-tagged-AXIN1 and nontransfected cells were used as negative controls. After FLAG-IP, endogenous E-cadherin and TCF7L2 were shown to bind equally to WT $\beta$-catenin and both variants. (C) The same $\beta$-catenin variants were cotransfected with Myc-tagged AXIN1 into HEK293 cells. All variants bind equally to AXIN1. (D) Cotransfection with GFP-APC in HEK293 cells. GFP-APC expresses a GFP-tagged murine APC fragment (amino acids 1199-2167) encoding all the 20-amino acid $\beta$-catenin-binding repeats and AXIN-binding domains. Both the K335I and N387K variants associate much more weakly to GFP-APC. In the latter 2 experiments, cotransfection of GFP-APC and Myc-AXIN1 was used as negative IP control. All transfections experiments were performed 3 times with identical results.

of APC-Ser1507 and forms an ionic bond with K335; however, it carries an apolar A688 at the position corresponding to pS1504 in APC (Supplementary Figure 5D). In AXIN, only hydrophobic residues are present at this interface (not shown), which form weak van der Waals contacts with the polar amine of K335 but might form more favorable contacts when the lysine is mutated to hydrophobic isoleucine. To summarize, K335 forms more energetically favorable interactions with APC than with any of the other proteins, explaining the observed relatively large effect of K355 mutation on APC binding specifically.

The N387K mutation will influence hydrogen bond formation between $\beta$-catenin and the peptide backbone of APC, LEF/TCF, and E-cadherin and is expected to reduce the strength of the interaction in all complexes (Supplementary Figures $5 E$ and $6 A$ and $B$ ). In this case, the structural 


\section{SNU449 AXIN1 repaired}

A

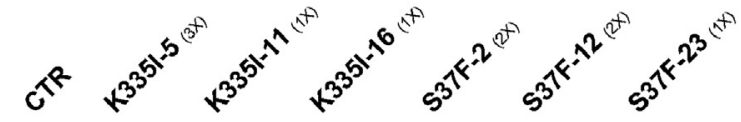

$\beta$-catenin

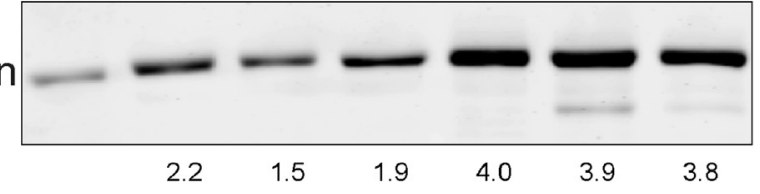

Tubulin

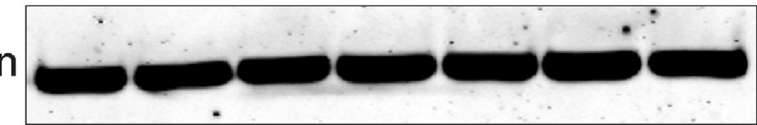

B
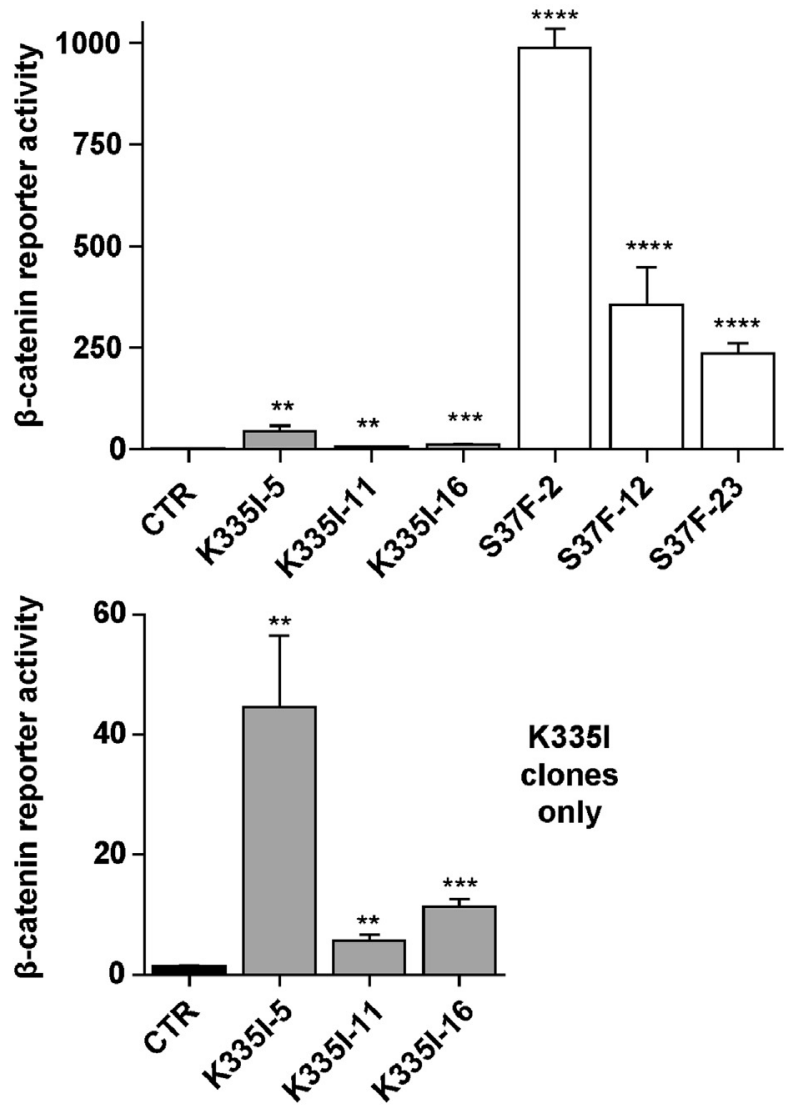

C

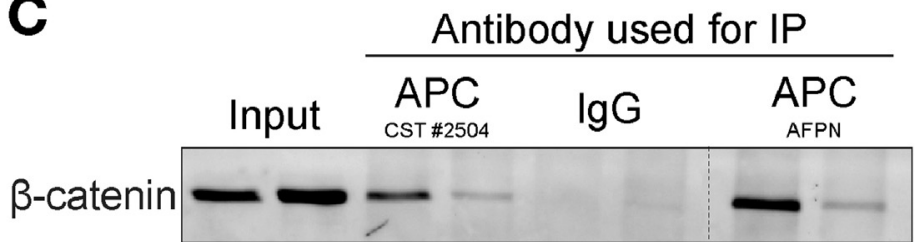

APC

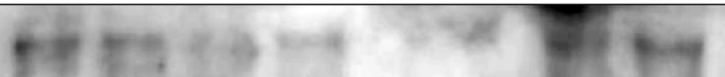

HEK293
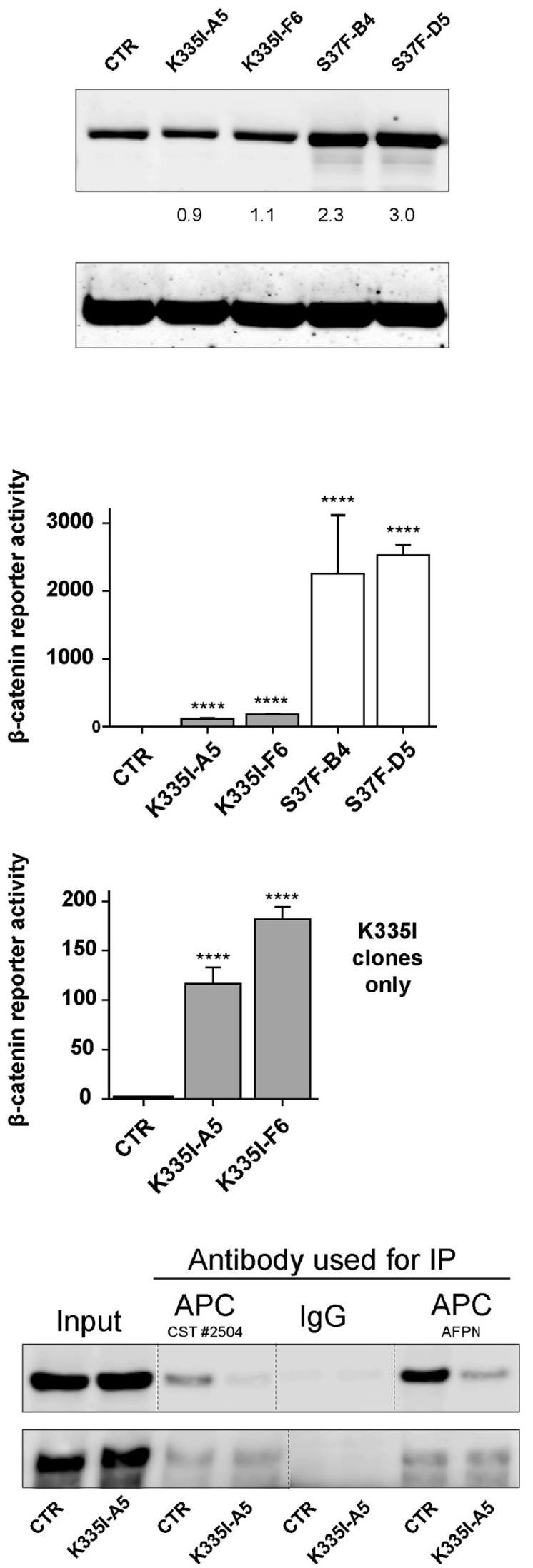
analysis provided no direct clues explaining the reduced binding of specifically APC to this variant.

\section{Selective Loss of APC Binding Is a Common Feature of Armadillo Repeat 5 and 6 Mutations}

Currently, more than 150 individual neoplasias have been identified carrying amino acid alterations in repeats $5 / 6$ of $\beta$ catenin (Figure 1 and Supplementary Table 1). Besides the K335 and N387 amino acid alterations, residues Y333, E334, R376, and especially W383 are also mutated in neoplasias regularly. Within the $\beta$-catenin structure, these residues are organized in 2 clusters in close proximity to each other (Figure $7 A$ ). We generated expression plasmids for these variants and tested them for binding to APC, TCF7L2, and Ecadherin. We also took along 1 commonly observed R582W variant $(\mathrm{n}=14)$ located toward the end of the armadillo repeats. As can be seen in Figure $7 B$ and $C$, most of these newly generated mutants show loss of APC binding, but all retain binding to TCF7L2 and E-cadherin. The only exceptions are the E334K and $\mathrm{R} 582 \mathrm{~W}$ variants, which behave identical to WT protein with respect to APC binding.

Next, we performed $\beta$-catenin reporter assays for all of the armadillo repeat 5/6 variants, taking S33Y and WT $\beta$ catenin along as controls (Figure $7 D$ ). All variants showing a reduced binding to APC, yield a 1.5-3-fold higher induction of reporter activity compared with the WT, whereas the E334K and R582W variants behave similar to the WT.

Importantly, analysis of the available protein structures shows that all additional mutated residues associated with increased signaling (Y333, R376, and W383), form direct interactions with APC (Figure $7 E$ and $F$ ). In fact, binding of cluster-1 residues (Figure $7 A$ and $E$ ) to APC creates an extensive network in which oppositely charged groups alternate and form multiple ionic interactions and hydrogen bonds with neighboring residues. Any mutation in this network will affect several highly optimized interactions, explaining the relatively large effect on APC binding that we observed. E334 does not form direct interactions with APC, possibly explaining its lack of increased signaling when mutated, although it may be involved in properly orienting the R376 residue.

In TCF and E-cadherin, the highly cooperative cluster-1 network is much reduced in size due to replacement of crucial serine sidechains (that are phosphorylated in APC) with hydrophobic residues (V44 in TCF and A688 in Ecadherin) (Supplementary Figure $6 C$ and $D$ ). These apolar residues cannot favorably interact with the polar sidechains in cluster-1, which means that the contribution of this cluster to the stability of TCF and cadherin complexes with $\beta$-catenin is weaker than for APC, explaining why cluster-1 mutations have a relatively smaller effect on their binding.
In cluster-2 (Figure $7 A$ and $F$ ), the sidechain of W383 forms van der Waals contacts with 2 threonine residues on APC, forming a small hydrophobic core that contributes to complex stability. Mutation of W383 will disrupt this energetically favorable region, resulting in loss of APC binding. TCF residues do not interact with the sidechain of W383 in $\beta$-catenin, and its mutation will not affect stability of the TCF complex (Supplementary Figure $6 E$ ). The situation is less clear in E-cadherin, in which favorable interactions between W383 from $\beta$-catenin and Y681 from E-cadherin are formed (Supplementary Figure 6F).

\section{Armadillo Repeat 5 and 6 Mutations Co-occur With Other $\beta$-Catenin Enhancing Mutations in Colorectal, but not in Hepatocellular, Carcinomas}

Mutations in the repeats 5 and 6 region of $\beta$-catenin have been mainly reported in liver, colon, and kidney tumors, ${ }^{5,8,28-31}$ and anecdotally in several other tumor types. The stronger activating $\beta$-catenin mutations encompassing exon 3 are known to occur in a mutually exclusive fashion with disease-causing APC mutations, ${ }^{32}$ showing that either mutation is sufficient to drive tumorigenesis. When we analyzed colorectal cancers carrying armadillo repeat $5 / 6$ mutations for concomitant defects in other genes linked to $\beta$-catenin signaling, these were observed in 11 out of 13 cancers (Supplementary Table 3). Seven out of 13 cancers carried $A P C$ mutations, but, interestingly, 3 out of 7 express a truncated APC protein that is expected to retain a significant level of $\beta$-catenin regulation that is 3 or more of the 20 AARs. ${ }^{4}$ Three other cancers carry truncating AXIN2 or RNF43 mutations that are generally also considered to be weak signaling activators. A similar analysis of 20 HCCs identified no accompanying $\beta$-catenin signaling activating, except for a single truncating ZNRF3 mutation (Supplementary Table 4). Thus, in contrast to liver cancers, the armadillo hotspot mutations observed in colorectal cancers are often accompanied by other weak mutations that may synergistically enhance $\beta$-catenin signaling to levels, supporting tumor growth.

\section{Discussion}

The $\beta$-catenin signaling pathway is one of the most commonly deregulated pathways among cancers. ${ }^{1}$ In colorectal cancers, this is predominantly accomplished by inactivating APC mutations, whereas in liver cancers, for example, aberrant activation has been mainly attributed to activating mutations in the CTNNB1 gene $(20 \%-25 \%)^{4,7,33-35}$ The exon 3-related $\beta$-catenin mutations acquire enhanced signaling activities by interfering

Figure 6. Endogenous expression in cell lines confirms increased signaling and reduced affinity for APC. (A) Introduction of the K335I and S37F mutation in SNU449 cells leads to a modest increase in total $\beta$-catenin protein levels. In HEK293 cells, this is restricted to S37F clones. Numbers in brackets refer to the number of CTNNB1 alleles expressing that variant. HEK293 clones are homozygous in all cases. $(B) \beta$-catenin luciferase reporter assay of all available clones. WRE/MRE ratios are shown. Data are presented as mean \pm standard deviation. ${ }^{\star \star} P<.01 ;{ }^{* \star \star} P<.001 ;{ }^{* \star \star \star} P<.0001$. The bottom half shows the K335I clones in comparison with the parental line. (C) IP of endogenous APC shows reduced binding of K335I. Two independent APC antibodies were used. Vertical dashed lines indicate removed irrelevant lanes. CTR, control; IgG, immunoglobulin G. 
with proper $\mathrm{N}$-terminal phosphorylation and subsequent proteolytic degradation. More recently, mutations in the armadillo repeat domains 5 and 6 of $\beta$-catenin have been identified in a substantial number of cancers, especially those of the liver. Here, we provide direct evidence that these mutants are potent inducers of HCC development in mice. In addition, we uncover a novel mode of $\beta$-catenin activation for these mutants, that is, they reduce the binding to APC while simultaneously retaining binding to TCF/LEF. The latter is important to allow sufficient enhancement of the $\beta$-catenin target genes driving tumor formation. A weaker binding to APC is expected to increase the signaling pool of $\beta$-catenin, enhancing the expression of $\beta$-catenin

A

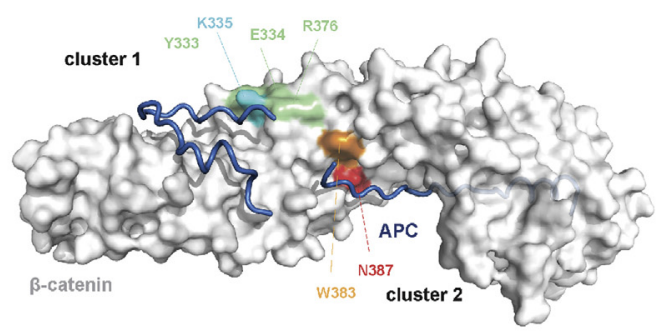

B
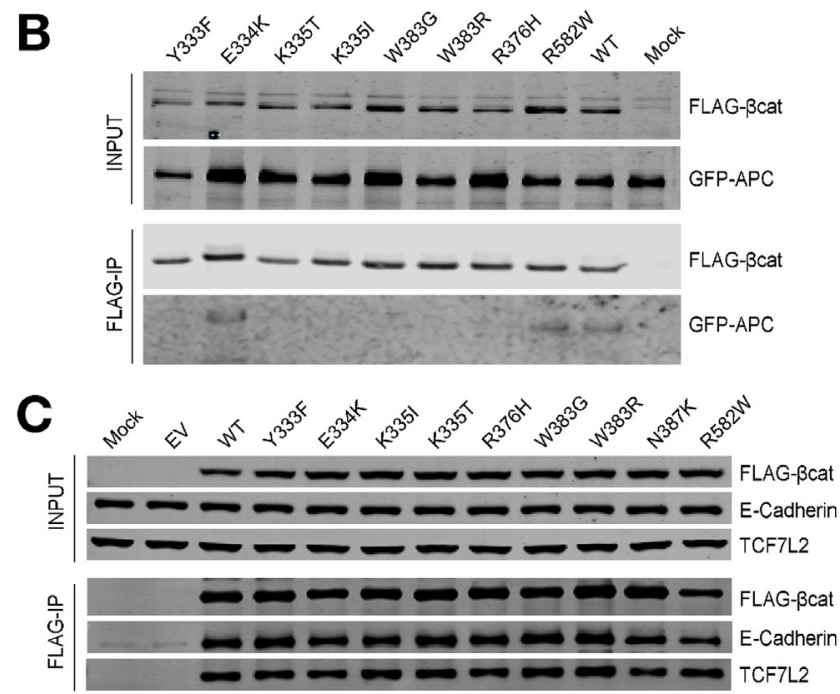

D

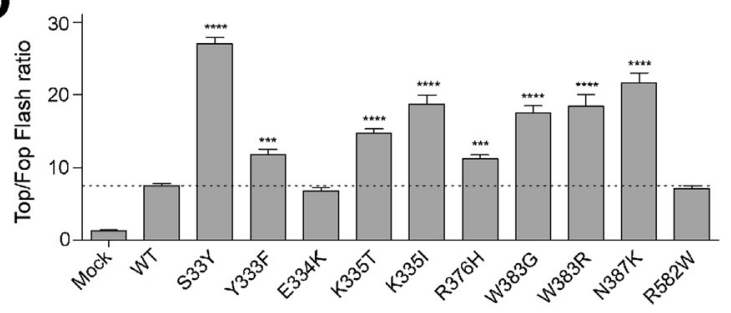

E

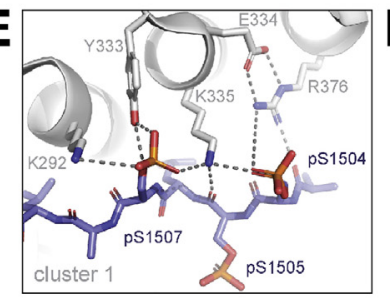

target genes driving tumor formation. However, if such a mutation would simultaneously reduce binding to the essential nuclear TCF/LEF transcriptional cofactors, the overall activation of target genes would not increase or even be reduced compared with WT $\beta$-catenin. In accordance with this, mutation of $\mathrm{K} 312$ and $\mathrm{K} 435$ residues is rarely observed, because these so-called charged buttons of $\beta$ catenin are essential for binding of most proteins to the armadillo superhelix by forming an ionic bond to conserved glutamate residues. ${ }^{2,36}$

When mapping the mutated residues on the protein structure of $\beta$-catenin, 2 clusters emerge that appear to be critical binding domains with APC (Figure 7A). Cluster-1 is centered around K335 and further involves residues Y333 and R376. Previously, these were all identified to directly interact with phosphorylated 20-amino acid repeats of APC but also, to some extent, with cadherins and TCF/LEF. ${ }^{25}$ However, the IP experiments show that their mutation selectively results in loss of APC binding, while leaving binding to TCF and E-cadherin unaffected. For the K335 residue itself, the structural analyses provide a rationale because it forms more energetically favorable interactions with APC than with any of the other proteins. Likewise, the structural analyses show that Y333 is not critical for TCF binding, whereas R376 is not important for cadherin binding. It is interesting to consider that an extensive ion-pair network is formed upon $\beta$-cateninAPC complex formation, while much smaller, fragmented networks are present in the TCF and E-cadherin complexes. Ion-pair networks are cooperative entities from which removal of a single residue through mutation will have a relatively large effect on the stability of the region. ${ }^{37-39}$ This

Figure 7. Increased signaling and selective reduction of APC binding for most armadillo repeat $5 / 6$ variants. $(A)$ Surface representation of $\beta$-catenin in gray with bound APC as tube representation in blue. K335 in cluster-1 is colored light blue, with surrounding residues Y333, E334, and R376 in light green. N387 in cluster-2 is colored red, and the other commonly mutated W383 is colored orange. (B) Cotransfection of indicated FLAG-tagged $\beta$-catenin variants with GFP-APC in HEK293 cells. After FLAG-IP, most of the commonly observed armadillo repeat $5 / 6$ variants associate much more weakly to GFP-APC. (C) FLAG-tagged $\beta$-catenin variants were transfected in HCT116 cells. After FLAG-IP, all variants are shown to bind equally to endogenous TCF7L2 and E-cadherin. (D) A TOP-FOPflash $\beta$-catenin reporter assay in HEK293 cells shows that all armadillo repeat $5 / 6$ variants that bind more weakly to APC show a 1.5-3-fold increased signaling compared with WT $\beta$-catenin. TOP-FOPflash ratios are shown. Experiments were performed in triplicate and repeated twice. Data are presented as mean \pm standard deviation. ${ }^{\star \star \star} P<.001 ;{ }^{\star \star \star \star} P<.0001$. (E) Details of the cluster-1 complex, with $\beta$-catenin in gray and APC in blue. A 6-residue ion-pair network is formed between E334-R376;R376pS1504;pS1504-K335;K335-pS1507;pS1507-K292. In fact, the network is even bigger and extends beyond K292 (not shown). Hydrogen bonds are indicated with dashed lines. (F) Details of the cluster-2 complex. W383 packs against the hydrophobic methyl-groups (b/ue carbons) of the sidechains of T1493 and T1496 and so forms favorable van der Waals interactions (curved lines). 
may explain why the mutation of K335 has a much larger effect on complex formation with APC (in which it is a central residue forming multiple ionic bonds) than on complex formation with TCF and E-cadherin (in which the lysine is a peripheral residue in the smaller network).

Cluster-2 involves residues W383 and N387. Previously, the W383 residue was already identified as important for APC binding because a W383A mutant failed to bind APC while retaining $\mathrm{LEF} / \mathrm{TCF} 7 \mathrm{~L} 2$ association, ${ }^{40}$ which is in accordance with the structural analyses, because TCF does not interact with W383. For the commonly mutated N387 residue, the structural analysis provided no direct clues explaining the reduced binding of, specifically, APC. Nevertheless, our IP and structural analyses provide clear evidence that both clusters are more relevant for APC binding than for TCF and cadherins.

Despite 2 decades of research, the exact mechanism through which APC functions in the destruction complex is still not fully understood. Several models not necessarily excluding each other have been proposed. ${ }^{41,42}$ One model proposes that APC is involved in sequestering $\beta$-catenin within the cytoplasm, thereby preventing it from entering the nucleus. ${ }^{43,44}$ Other models suggest that APC protects S/ $\mathrm{T}$ phosphorylated $\beta$-catenin from dephosphorylation by PP2A, that it may be required to interface $\beta$-catenin with the ubiquitin and proteasome machinery, or that it is required to displace AXIN from phosphorylated $\beta$-catenin to allow a new cycle of $\beta$-catenin breakdown. ${ }^{24,45,46}$ Whichever model turns out to be true, in all cases, a weaker association of mutant $\beta$-catenin with APC will make the process less efficient and effectively lead to more signaling.

How to reconcile the weak APC/mutant $\beta$-catenin interaction with the rather modest increased signaling activity associated with these variants? Current models of APC's contribution to the destruction complex propose that direct binding of $\beta$-catenin to APC is not absolutely required for a proper level of $\beta$-catenin turnover, as long as APC is present within the destruction complex-for example, through binding to AXIN. ${ }^{41,42,44,47}$ This partial functional redundancy between APC and AXIN is most likely also responsible for keeping the signaling activity of the armadillo repeats $5 / 6$ mutants in check, although it will be less efficient when compared with WT $\beta$-catenin.

Mutations in this region of $\beta$-catenin have been mainly reported in liver cancers, Wilms tumors, and tumors of the large intestine, ${ }^{5,8,28-31}$ and anecdotally in several other tumor types, but their true frequency is probably underestimated because, in the past, CTNNB1 mutation analysis was largely restricted to the exon 3 hotspot. Therefore, whole-exome or -genome mutational analyses that are becoming more routine procedures for tumor investigations nowadays are likely to more frequently uncover these specific mutations. Given their weak activation nature, these mutations are more likely going to be identified in tumor types that require a minimal to moderate activation of the pathway to support their growth. ${ }^{4,48,49}$ In that respect, their presence in colorectal cancer is somewhat surprising because these cancers generally select for higher $\beta$-catenin signaling levels. ${ }^{4}$ Our analysis shows, however, that in colorectal cancer, the armadillo hotspot mutations are often accompanied by other (weak) mutations that synergistically may enhance $\beta$-catenin signaling to levels supporting tumor growth. A similar scenario may be at work for Wilms tumors, in which the armadillo repeat 5/6 mutations often associate with those of WTX (official name, AMER1), ${ }^{31}$ also leading to a modest signaling enhancement. In liver cancers, however, the weak signaling associated with these armadillo repeat variants appears to be sufficient to support tumor growth because they are not clearly associated with defects in other Wnt/ $\beta$-catenin signaling-related components.

This latter observation is also in line with our tumor induction experiments in mice and the analysis of SNU449 and HEK293 cell lines with knock-in mutations. In a direct comparison, endogenous levels of the K335I mutant are clearly more potent inducers of $\beta$-catenin signaling than WT $\beta$-catenin, but they are rather weak in comparison with the strong S37F mutation. Nevertheless, within 6-7 weeks after injection, they lead to a massive HCC formation, which is comparable in timeframe with the S45Y and $\Delta 90 \mathrm{~N}-\beta$-catenin variants. ${ }^{17}$ However, immunohistochemical analysis showed no clear nuclear accumulation for the K335I and N387K variants. Similar observations have been made for K335/N387 mutant human liver tumors, which also lack a clear nuclear $\beta$-catenin accumulation associated with a weak activation of target genes. ${ }^{8}$ Combined, these results show that a strong nuclear presence is no prerequisite for efficient liver cancer formation. In support of this, we have recently shown that liver specific loss of AXIN1 requires $\beta$ catenin to induce hepatocarcinogenesis, which, however, was associated with only a weak activation of $\beta$-catenin target genes. ${ }^{18}$ Buchert et $\mathrm{al}^{48}$ have shown that late-onset hepatocellular tumors were present in all mice carrying a hypomorphic APC mutation associated with just a modest increase in $\beta$-catenin signaling, and tumor formation was absent or largely prevented with slightly increased or decreased signaling. All of these examples show that only modest increases in $\beta$-catenin signaling may nevertheless be relevant for hepatocellular tumorigenesis.

In conclusion, our molecular and structural analyses uncover a novel mutational mechanism of enhanced $\beta$-catenin signaling. In contrast to the $\mathrm{N}$-terminal mutations in $\beta$-catenin that directly impair its phosphorylation by GSK3/CK1 $\alpha$ or binding to $\beta$ - TrCP, the hotspot mutations within armadillo repeats 5 and 6 lead to enhanced signaling through reduced binding to APC while simultaneously retaining the interaction with their nuclear TCF/LEF transcriptional cofactors. The latter is important to allow sufficient enhancement of $\beta$ catenin target genes driving tumor formation. A second difference with the N-terminal mutants appears to be that they often co-occur with other mutations affecting $\beta$-catenin signaling, at least in colorectal and Wilms tumors.

\section{Supplementary Material}

Note: To access the supplementary material accompanying this article, visit the online version of Gastroenterology at www.gastrojournal.org, and at https://doi.org/10.1053/ j.gastro.2019.11.302. 


\section{References}

1. Zhan T, Rindtorff N, Boutros M. Wht signaling in cancer. Oncogene 2017;36:1461-1473.

2. Valenta $T$, Hausmann $G$, Basler K. The many faces and functions of $\beta$-catenin. EMBO J 2012;31:2714-2736.

3. Nusse $\mathrm{R}$, Clevers $\mathrm{H}$. Wnt/ $\beta$-catenin signaling, disease, and emerging therapeutic modalities. Cell 2017;169: 985-999.

4. Albuquerque C, Bakker ER, van Veelen W, et al. Colorectal cancers choosing sides. Biochim Biophys Acta Rev Cancer 2011;1816:219-231.

5. Pilati C, Letouze E, Nault JC, et al. Genomic profiling of hepatocellular adenomas reveals recurrent FRKactivating mutations and the mechanisms of malignant transformation. Cancer Cell 2014;25:428-441.

6. The Cancer Genome Atlas Research Network, Kandoth C, Schultz N, et al. Integrated genomic characterization of endometrial carcinoma. Nature 2013; 497(7447):67-73.

7. The Cancer Genome Atlas Research Network. Comprehensive and integrative genomic characterization of hepatocellular carcinoma. Cell 2017;169:1327-1341.

8. Rebouissou S, Franconi A, Calderaro J, et al. Genotype-phenotype correlation of CTNNB1 mutations reveals different $\beta$-catenin activity associated with liver tumor progression. Hepatology 2016;64:2047-2061.

9. Wang Z, Vogelstein B, Kinzler KW. Phosphorylation of $\beta$-catenin at $\mathbf{S} 33, \mathbf{S} 37$, or $\mathrm{T} 41$ can occur in the absence of phosphorylation at T45 in colon cancer cells. Cancer Res 2003;63:5234-5235.

10. Forbes SA, Beare D, Gunasekaran P, et al. COSMIC: exploring the world's knowledge of somatic mutations in human cancer. Nucleic Acids Res 2015;43(D1):D805-D811.

11. Li CM, Kim CE, Margolin AA, et al. CTNNB1 mutations and overexpression of $\mathrm{Wnt} / \beta$-catenin target genes in WT1-mutant Wilms' tumors. Am J Pathol 2004; 165:1943-1953.

12. Colnot $\mathbf{S}$. Focusing on $\beta$-catenin activating mutations to refine liver tumor profiling. Hepatology 2016;64: 1850-1852.

13. Dubbink HJ, Hollink I, Avenca Valente C, et al. A novel tissue-based $\beta$-catenin gene and immunohistochemical analysis to exclude familial adenomatous polyposis among children with hepatoblastoma tumors. Pediatr Blood Cancer 2018;65(6):e26991.

14. Deng W, Vanderbilt DB, Lin CC, et al. SOX9 inhibits $\beta$-TrCP-mediated protein degradation to promote nuclear GLI1 expression and cancer stem cell properties. J Cell Sci 2015;128:1123-1138.

15. Zeng L, Fagotto F, Zhang T, et al. The mouse Fused locus encodes Axin, an inhibitor of the Wnt signaling pathway that regulates embryonic axis formation. Cell 1997; $90: 181-192$.

16. Tao J, Xu E, Zhao Y, et al. Modeling a human hepatocellular carcinoma subset in mice through coexpression of met and point-mutant $\beta$-catenin. Hepatology 2016; 64:1587-1605.

17. Qiao $Y, X u M, T a o ~ J$, et al. Oncogenic potential of $\mathrm{N}$-terminal deletion and $\mathrm{S} 45 \mathrm{Y}$ mutant $\beta$-catenin in promoting hepatocellular carcinoma development in mice. BMC Cancer 2018;18:1093.

18. Qiao Y, Wang J, Karagoz E, et al. Axis inhibition protein 1 (Axin1) deletion-induced hepatocarcinogenesis requires intact $\beta$-catenin but not Notch cascade in mice. Hepatology 2019;70:2003-2017.

19. Adebayo Michael AO, Ko S, Tao J, et al. Inhibiting glutamine-dependent mTORC1 activation ameliorates liver cancers driven by $\beta$-catenin mutations. Cell Metab 2019;29:1135-1150.

20. Wang W, Xu L, Liu P, et al. Blocking Wnt secretion reduces growth of hepatocellular carcinoma cell lines mostly independent of $\beta$-catenin signaling. Neoplasia 2016;18:711-723.

21. Chen X, Calvisi DF. Hydrodynamic transfection for generation of novel mouse models for liver cancer research. Am J Pathol 2014;184:912-923.

22. Graham TA, Weaver C, Mao F, et al. Crystal structure of a $\beta$-catenin/Tcf complex. Cell 2000;103:885-896.

23. Huber AH, Weis WI. The structure of the $\beta$-catenin/Ecadherin complex and the molecular basis of diverse ligand recognition by $\beta$-catenin. Cell 2001;105:391-402.

24. Xing Y, Clements WK, Kimelman D, et al. Crystal structure of a $\beta$-catenin/Axin complex suggests a mechanism for the $\beta$-catenin destruction complex. Genes Dev 2003; 17:2753-2764.

25. Xing Y, Clements WK, Le Trong I, et al. Crystal structure of a $\beta$-catenin/APC complex reveals a critical role for APC phosphorylation in APC function. Mol Cell 2004; 15:523-533.

26. Rubinfeld B, Albert I, Porfiri E, et al. Binding of GSK3 $\beta$ to the APC- $\beta$-catenin complex and regulation of complex assembly. Science 1996;272(5264):1023-1026.

27. Liu J, Xing Y, Hinds TR, et al. The third 20 amino acid repeat is the tightest binding site of APC for $\beta$-catenin. J Mol Biol 2006;360:133-144.

28. Gadd S, Huff V, Walz AL, et al. A Children's Oncology Group and TARGET initiative exploring the genetic landscape of Wilms tumor. Nat Genet 2017;49: 1487-1494.

29. The Cancer Genome Atlas Network. Comprehensive molecular characterization of human colon and rectal cancer. Nature 2012;487(7407):330-337.

30. Yaeger R, Chatila WK, Lipsyc MD, et al. Clinical sequencing defines the genomic landscape of metastatic colorectal cancer. Cancer Cell 2018;33:125-136.

31. Perotti D, Hohenstein P, Bongarzone I, et al. Is Wilms tumor a candidate neoplasia for treatment with WNT/ $\beta$ catenin pathway modulators?-a report from the renal tumors Biology-Driven Drug Development Workshop. Mol Cancer Ther 2013;12:2619-2627.

32. Morin PJ, Kinzler KW, Sparks AB. $\beta$-catenin mutations: insights into the APC pathway and the power of genetics. Cancer Res 2016;76:5587-5589.

33. Zucman-Rossi J, Villanueva A, Nault JC, et al. Genetic landscape and biomarkers of hepatocellular carcinoma. Gastroenterology 2015;149:1226-1239.

34. Dahmani R, Just PA, Perret C. The Wnt/ $\beta$-catenin pathway as a therapeutic target in human hepatocellular 
carcinoma. Clin Res Hepatol Gastroenterol 2011;35: 709-713.

35. Wang W, Pan Q, Fuhler GM, et al. Action and function of Wnt/ $\beta$-catenin signaling in the progression from chronic hepatitis C to hepatocellular carcinoma. J Gastroenterol 2017;52:419-431.

36. Xu W, Kimelman D. Mechanistic insights from structural studies of $\beta$-catenin and its binding partners. J Cell Sci 2007;120:3337-3344.

37. Lebbink JH, Knapp S, van der Oost J, et al. Engineering activity and stability of Thermotoga maritima glutamate dehydrogenase. II: construction of a 16-residue ion-pair network at the subunit interface. J Mol Biol 1999; 289:357-369.

38. Lebbink JH, Consalvi V, Chiaraluce R, et al. Structural and thermodynamic studies on a salt-bridge triad in the NADP-binding domain of glutamate dehydrogenase from Thermotoga maritima: cooperativity and electrostatic contribution to stability. Biochemistry 2002;41: 15524-15535.

39. Karshikoff A, Nilsson L, Ladenstein R. Rigidity versus flexibility: the dilemma of understanding protein thermal stability. FEBS J 2015;282:3899-3917.

40. von Kries JP, Winbeck G, Asbrand C, et al. Hot spots in $\beta$-catenin for interactions with LEF-1, conductin and APC. Nat Struct Biol 2000;7:800-807.

41. Stamos JL, Weis WI. The $\beta$-catenin destruction complex. Cold Spring Harb Perspect Biol 2013;5(1):a007898.

42. van Kappel EC, Maurice MM. Molecular regulation and pharmacological targeting of the $\beta$-catenin destruction complex. Br J Pharmacol 2017;174:4575-4588.

43. Ha NC, Tonozuka T, Stamos JL, et al. Mechanism of phosphorylation-dependent binding of APC to $\beta$-catenin and its role in $\beta$-catenin degradation. Mol Cell 2004; 15:511-521.

44. Roberts DM, Pronobis MI, Poulton JS, et al. Deconstructing the $\beta$-catenin destruction complex: mechanistic roles for the tumor suppressor APC in regulating Wnt signaling. Mol Biol Cell 2011;22:1845-1863.

45. Su Y, Fu C, Ishikawa S, et al. APC is essential for targeting phosphorylated $\beta$-catenin to the $\mathrm{SCF}^{\beta-\operatorname{TrCP}}$ ubiquitin ligase. Mol Cell 2008;32:652-661.
46. Pronobis MI, Rusan NM, Peifer M. A novel GSK3regulated APC:Axin interaction regulates Wnt signaling by driving a catalytic cycle of efficient $\beta$ catenin destruction. Elife 2015;4:e08022.

47. Pronobis MI, Deuitch N, Posham V, et al. Reconstituting regulation of the canonical Wnt pathway by engineering a minimal $\beta$-catenin destruction machine. Mol Biol Cell 2017;28:41-53.

48. Buchert M, Athineos D, Abud HE, et al. Genetic dissection of differential signaling threshold requirements for the $\mathrm{Wnt} / \beta$-catenin pathway in vivo. PLoS Genet 2010;6(1):e1000816.

49. Bakker ER, Hoekstra E, Franken PF, et al. $\beta$-catenin signaling dosage dictates tissue-specific tumor predisposition in Apc-driven cancer. Oncogene 2013;32: 4579-4585.

\section{Author names in bold designate shared co-first authorship.}

Received September 9, 2019. Accepted November 11, 2019.

\section{Correspondence}

Address correspondence to: Ron Smits, PhD, Erasmus MC, Department of Gastroenterology and Hepatology, Room Na-1011, Wytemaweg 80, 3015 CN Rotterdam, The Netherlands. e-mail: m.j.m.smits@erasmusmc.nl; fax: $(+31)$ 107032793.

\section{Acknowledgments}

Author contributions: Pengyu Liu performed the majority of experimental work and data analysis and authored the manuscript; Binyong Liang, Manning Qian, and Xin Chen performed and coordinated the animal experiments involving hydrodynamic transfection; Menggang Liu, Marla Lavrijsen, and Shan Li assisted with the experiments; Joyce H.G. Lebbink performed all the structural protein analyses and authored parts of the manuscript; Maikel $P$ Peppelenbosch supervised the project and improved the manuscript; and Ron Smits coordinated the project and authored the manuscript. All authors reviewed the results and approved the final version of the manuscript. We thank Dr Peter Hohenstein (Leiden University Medical Center, The Netherlands) for critical reading of the manuscript.

Conflicts of interest

The authors disclose no conflicts.

Funding

This research was financially supported by a China Scholarship Council PhD fellowship to Pengyu Liu (file no. 20140822 0029), Shan Li (file no. 2014 0806 0053) and Manning Qian (file no. 20180832 0464) and a National Institutes of Health grant (R01CA204586) to Xin Chen. Joyce Lebbink is supported by the gravitation program $(024.001 .028)$ from The Netherlands Organization for Scientific Research (NWO). 


\section{Supplemental Methods and Reagents}

\section{Additional Methods for Analysis of Mouse Livers and Tumors: H\&E Staining and Immunohistochemistry}

Mouse liver tissues were fixed in $4 \%$ paraformaldehyde overnight at $4^{\circ} \mathrm{C}$ for subsequent paraffinembedding. Sections were cut at $5-\mu \mathrm{m}$ thickness and then used for H\&E staining and immunohistochemistry. H\&E staining was performed to determine characteristics of neoplastic foci. For immunohistochemistry, antigen retrieval was performed in sodium citrate buffer $(\mathrm{pH}$ 6.0 ) by placement in a microwave on high for $10 \mathrm{mi}-$ nutes, followed by a 20-minute cooldown at room temperature. After a blocking step with the 5\% goat serum and Avidin-Biotin blocking kit (Vector Laboratories, Burlingame, CA), the slides were incubated with primary antibodies (Supplementary Table 5) overnight at $4^{\circ} \mathrm{C}$. Slides were then subjected to 3\% hydrogen peroxide for 10 minutes to quench endogenous peroxidase activity. Subsequently, the biotin conjugated secondary antibody (Life Technology, Waltham, MA) was applied at a 1:500 dilution for 30 minutes at room temperature. Immunostainings were visualized using the Vectastain Elite $\mathrm{ABC}$ Kit (Vector Laboratories) using 3,3'-diaminobenzidine tetra hydrochloride as the substrate (Dako North America, Carpinteria, CA). Slides were counterstained with hematoxylin.

\section{RNA Extraction and Real-Time qRT-PCR}

Total mRNA was extracted from mouse liver tissues using the Quick RNA miniprep kit (Zymo Research, Irvine, CA). Complementary DNA was generated using the $5 \times$ iScript RT Supermix (Bio-Rad, Hercules, CA), according to the manufacturer's instructions. Messenger RNA (mRNA) expression was determined by qRT-PCR using SYBR Green Master Mix (Applied Biosystems, Foster City, CA) in an QuantStudio 6 Flex system (Applied Biosystems, Waltham, MA). Expression of each specific gene mRNA was normalized with the $18 \mathrm{~S}$ ribosomal RNA. Thermal cycling conditions included an initial hold period at $95^{\circ} \mathrm{C}$ for 10 minutes, which was followed by a 3-step PCR program of $95^{\circ} \mathrm{C}$ for 15 seconds, $60^{\circ} \mathrm{C}$ for 1 minute, and $72^{\circ} \mathrm{C}$ for 30 seconds, for a total of 40 cycles. Primers used in this study are shown in Supplementary Table 6.

\section{Cell Culture}

HEK293, HCT116, and SNU449 cells and all their derivatives were maintained in Dulbecco's modified Eagle medium (Lonza, Breda, The Netherlands) supplemented with $10 \%$ fetal bovine serum (Sigma-Aldrich). Identity of cell lines was confirmed by short tandem repeats (STR) genotyping, and all lines were mycoplasma free.
Clustered Regularly Interspaced Short

Palindromic Repeats/Cas9-Mediated Knock-In of CTNNB1 Mutations

Single guide RNAs (sgRNAs) were cloned into pSpCas9(BB)-2A-GFP (PX458), a gift from Feng Zhang (Addgene plasmid \#48138) ${ }^{1}$ using standard procedures. Single-stranded oligodeoxynucleotides (ssODNs) were designed following the guidelines proposed by Richardson et $\mathrm{al}^{2}$ and were ordered as Ultramer from Integrated DNA Technologies (Coralville, IA). Both the sgRNAs and ssODNs are described in Supplementary Table 8. Transfections were performed with the Amaxa Cell Line Nucleofector Kit-V (Lonza, Basel, Switzerland) and Nucleofector IIb device, according to the manufacturer's instructions. In brief, $5 \times$ $10^{6}$ cells were cotransfected with $2 \mu \mathrm{g}$ PX458 and 1-2 $\mu \mathrm{g}$ ssODN, using program T28 for SNU449 and Q1 for HEK293. After nucleofection, complete Dulbecco's modified Eagle medium with $7.5 \mu \mathrm{mol} / \mathrm{L}$ RAD51-stimulatory compound-1 (RS-1, Sigma-Aldrich) was used for cell culture. After 48 hours, GFP-positive cells were sorted by FACS and seeded as single cells in 96-well plates. DNA from clones grown successfully were isolated using the QuickExtract DNA Extraction Solution (Epicentre, Madison, WI). Each clone was prescreened using PCRs selectively amplifying either the WT or correctly altered sequence, according to the method described by Harayama et al. ${ }^{3}$ Candidate clones were subjected to Sanger sequencing and correctly modified clones were confirmed by next-generation sequencing on the Ion Torrent Personal Genome Machine System using amplicon fusion primers.

\section{Details of Immunoprecipitation Experiments}

For the IP experiments using expression plasmids, HEK293 or HCT116 cells were seeded in 6-well plates before transfection. When reaching $60 \%-80 \%$ confluence, 1 $\mu \mathrm{g}$ N-terminal FLAG-tagged $\beta$-catenin variant plasmids were cotransfected with either $1 \mu \mathrm{g}$ of APC-, AXIN-, or $\beta \mathrm{TrCP}$ expression plasmids by using FuGENE HD. After 24 hours, cells were washed by cold phosphate-buffered saline 2 times, and then cold $500 \mu \mathrm{L}$ lysis buffer was added to each well for 15 minutes. Our main lysis buffer consisted of 30 mmol/L Tris-Cl pH 7.4, 1\% Triton X-100, $150 \mathrm{mmol} / \mathrm{L} \mathrm{NaCl}$, $5 \mathrm{mmol} / \mathrm{L}$ EDTA, and $5 \mathrm{mmol} / \mathrm{L} \mathrm{NaF}$. For the $\beta$-TrCP coimmunoprecipitation experiments, we used the following lysis buffer: $10 \mathrm{mmol} / \mathrm{L}$ HEPES pH 7.9, $100 \mathrm{mmol} / \mathrm{L} \mathrm{NaCl}$, $1.5 \mathrm{mmol} / \mathrm{L} \mathrm{MgCl}_{2}, \quad 0.1 \% \mathrm{NP} 40$. The $\beta$-TrCP $/ \beta$-catenin interaction is short lived because of the rapid turnover by the proteasome, se we applied 2-hour proteasome inhibition using MG132 before precipitation. To all lysis steps, Halt Protease and Phosphatase Inhibitor Cocktail (Thermo Fisher Scientific) was added according to the manufacturer's instructions. Cells were collected by scraping, and lysate was cleared at $4^{\circ} \mathrm{C}$ by centrifugation at $11,000 \mathrm{~g}$ for 15 minutes. From the cleared lysate, $10 \%$ was taken as input control, to which the same volume of $2 \times$ Laemmli/ 
dithiothreitol (DTT) was directly added, followed by heating for 5 minutes at $95^{\circ} \mathrm{C}$. To the remainder of the supernatant, we added $10 \mu \mathrm{L}$ prewashed ANTI-FLAG M2 Affinity Gel (Sigma-Aldrich), followed by incubation at $4^{\circ} \mathrm{C}$ for 2 hours. Next, FLAG-beads were centrifuged and washed with lysis buffer for 3 times. Finally, the pellet was lysed in $50 \mu \mathrm{L} 2 \times$ Laemmli sample buffer with $0.1 \mathrm{~mol} / \mathrm{L}$ DTT and heated.

For the IP experiments using knock-in cell lines, cells were seeded in $15-\mathrm{cm}$ round culture plates. When nearly confluent, cells were washed by cold phosphate-buffered saline 2 times, and then 1.5-2 mL cold lysis buffer with protease/phosphatase inhibitors was added to each well for 15-30 minutes. Cells were collected by scraping, and lysate was cleared at $4{ }^{\circ} \mathrm{C}$ by centrifugation at $11,000 \mathrm{~g}$ for $15 \mathrm{mi}$ nutes. From the cleared lysate, $5 \%-10 \%$ was taken as input control, to which the same volume of $2 \times$ Laemmli/DTT was directly added, followed by heating for 5 minutes at $70^{\circ} \mathrm{C}$. To the cleared lysates, $5 \mu \mathrm{L}$ of the antibodies detecting APC or $\beta$-catenin was added (Supplementary Table 9). In addition, APC was immunoprecipitated using $20 \mu \mathrm{L}$ of the AFPN antibody. ${ }^{4}$ After 2 hours of incubation at $4^{\circ} \mathrm{C}, 25 \mu \mathrm{L}$ of Protein A/G magnetic beads (Pierce, Thermo Fisher Scientific) were added and incubated for an additional 2 hours. IPs were washed 3-4 times with lysis buffer and finally lysed in $80 \mu \mathrm{L} 2 \times$ Laemmli sample buffer with $0.1 \mathrm{~mol} / \mathrm{L}$ DTT, and heated at $70^{\circ} \mathrm{C}$ for 5 minutes. For detecting the
$\beta$-catenin/TCF7L2 interaction, we used the following buffer: $30 \mathrm{mmol} / \mathrm{L}$ Tris-Cl pH 7.4, 0.1\% Triton X-100, $150 \mathrm{mmol} / \mathrm{L}$ $\mathrm{NaCl}, 5 \mathrm{mmol} / \mathrm{L}$ EDTA, and $5 \mathrm{mmol} / \mathrm{L} \mathrm{NaF}$. For detecting the $\mathrm{APC} / \beta$-catenin interaction we used $25 \mathrm{mmol} / \mathrm{L}$ Tris-Cl $\mathrm{pH}$ 7.4, 1\% NP-40, $150 \mathrm{mmol} / \mathrm{L} \mathrm{NaCl}, 1 \mathrm{mmol} / \mathrm{L}$ EDTA, and 5\% glycerol. Alternatively, this buffer was used: $30 \mathrm{mmol} / \mathrm{L}$ Tris-Cl pH 7.4, 1\% Triton X-100, $300 \mathrm{mmol} / \mathrm{L} \mathrm{NaCl,} 5 \mathrm{mmol} /$ L EDTA, and $5 \mathrm{mmol} / \mathrm{L} \mathrm{NaF}$.

\section{Supplementary References}

1. Ran FA, Hsu PD, Wright J, et al. Genome engineering using the CRISPR-Cas9 system. Nat Protoc 2013; 8:2281-2308.

2. Richardson CD, Ray GJ, DeWitt MA, et al. Enhancing homology-directed genome editing by catalytically active and inactive CRISPR-Cas9 using asymmetric donor DNA. Nat Biotechnol 2016;34:339-344.

3. Harayama T, Riezman H. Detection of genome-edited mutant clones by a simple competition-based PCR method. PLoS One 2017;12(6):e0179165.

4. Smits R, Kielman MF, Breukel C, et al. Apc1638T: a mouse model delineating critical domains of the adenomatous polyposis coli protein involved in tumorigenesis and development. Genes Dev 1999;13:1309-1321. 

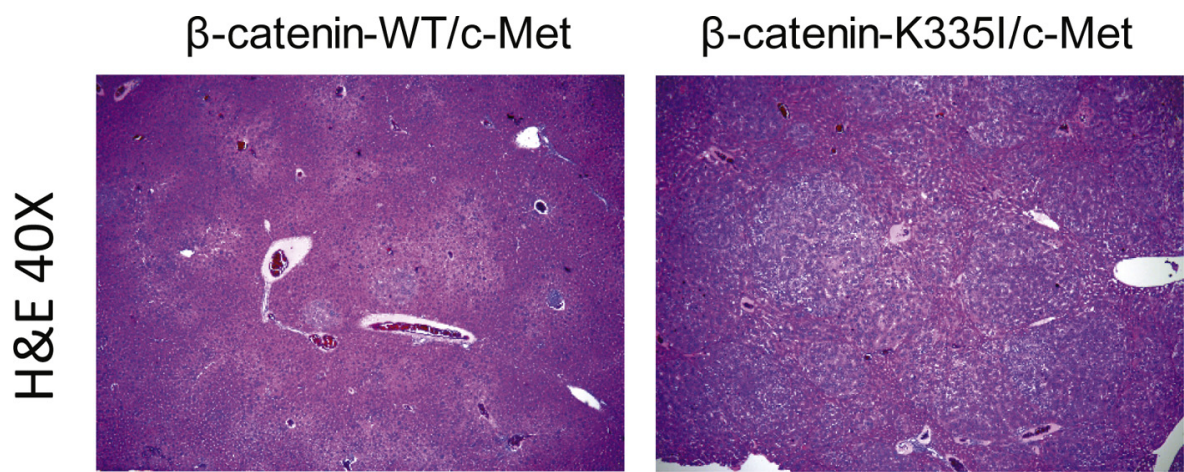

\section{$\beta$-catenin-N387K/c-Met}
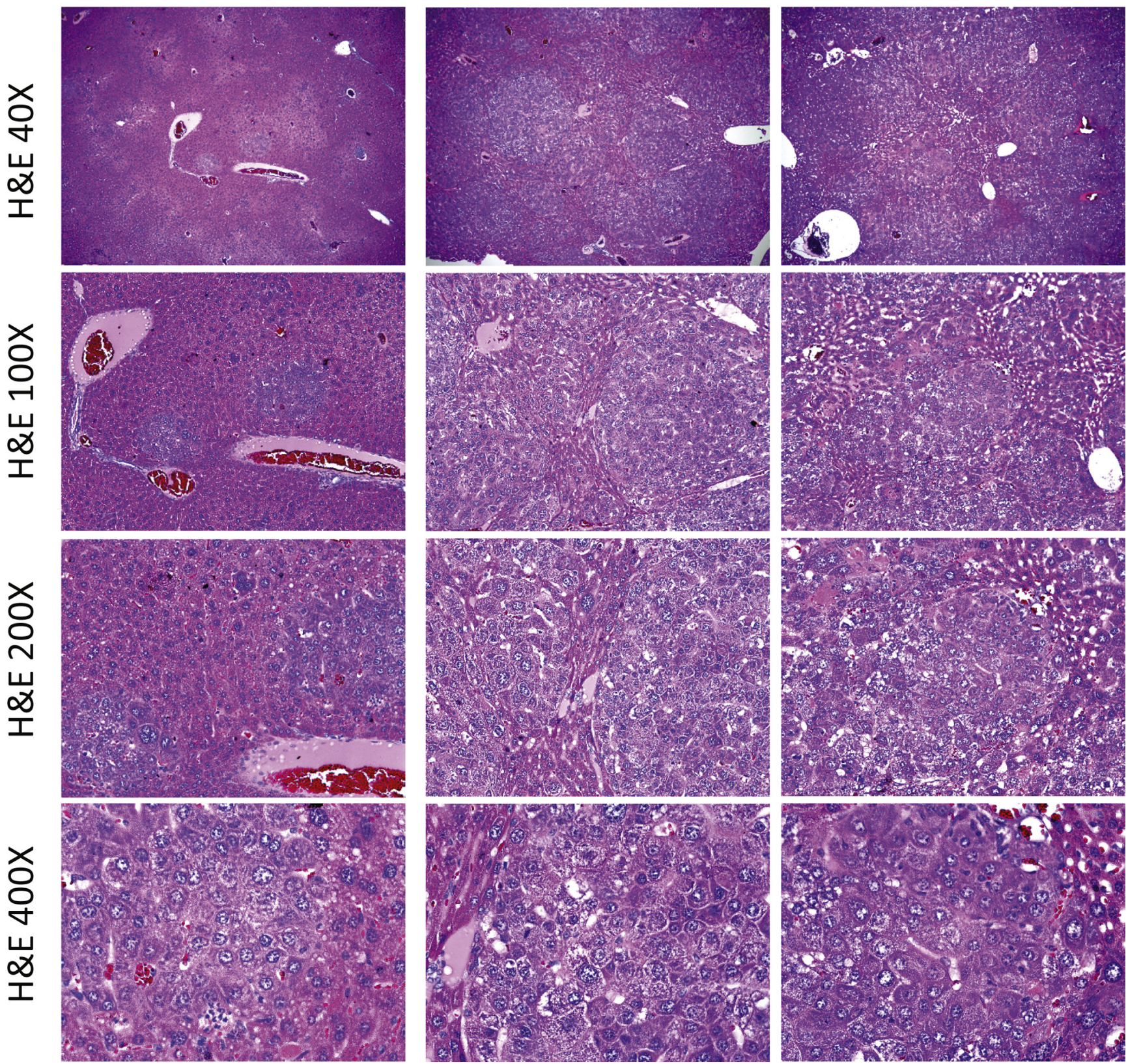

Supplementary Figure 1. H\&E stainings of representative HCC foci induced after hydrodynamic transfections at 4 different magnifications. Original magnifications are shown. 

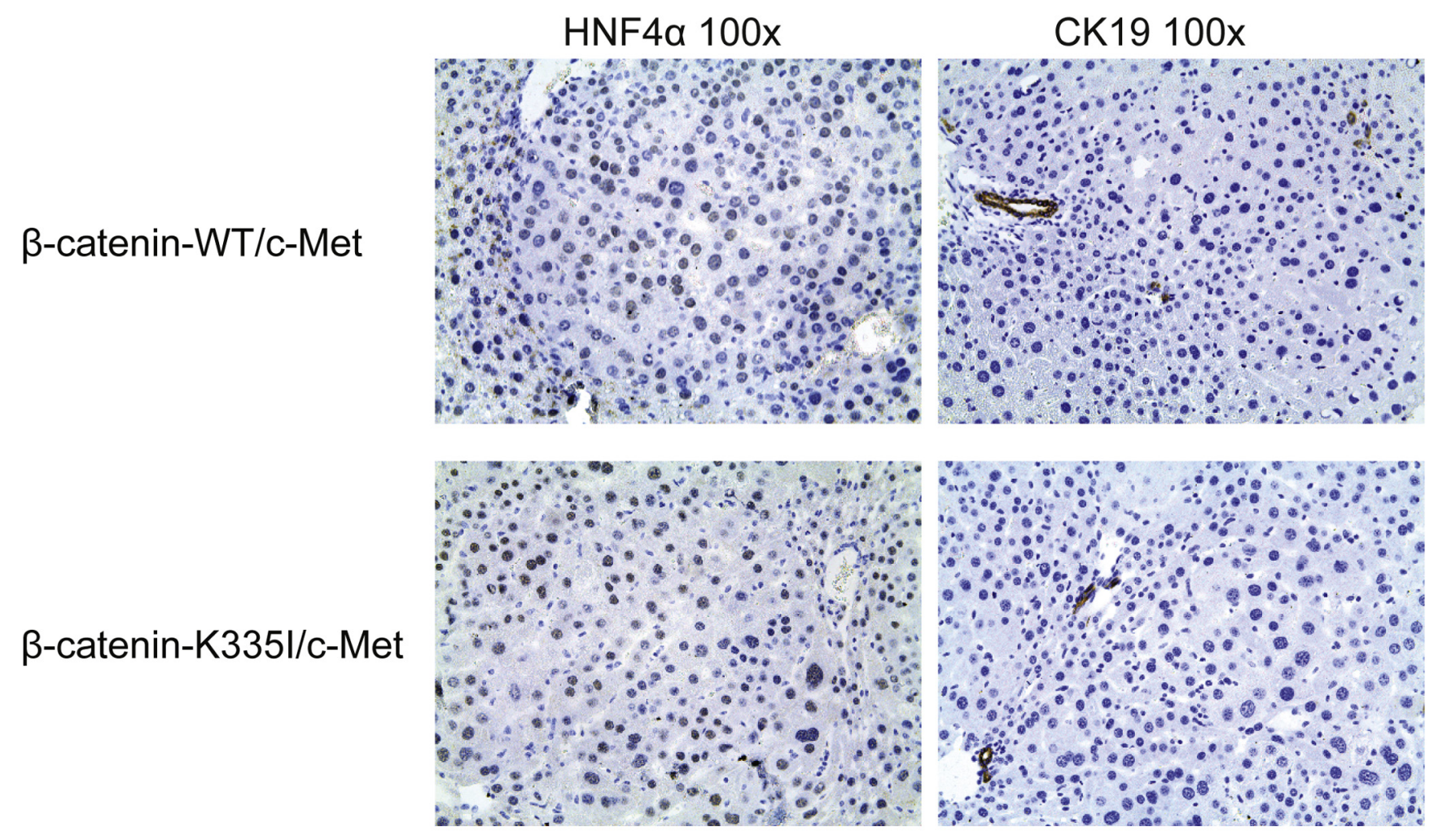

$\beta$-catenin-N387K/c-Met
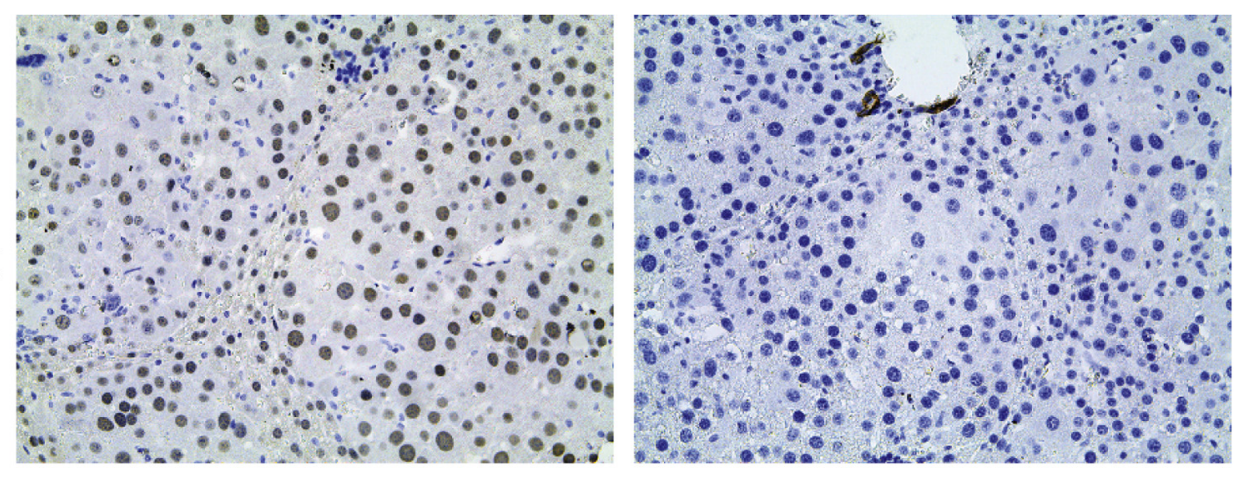

$\beta$-catenin-S45Y/c-Met
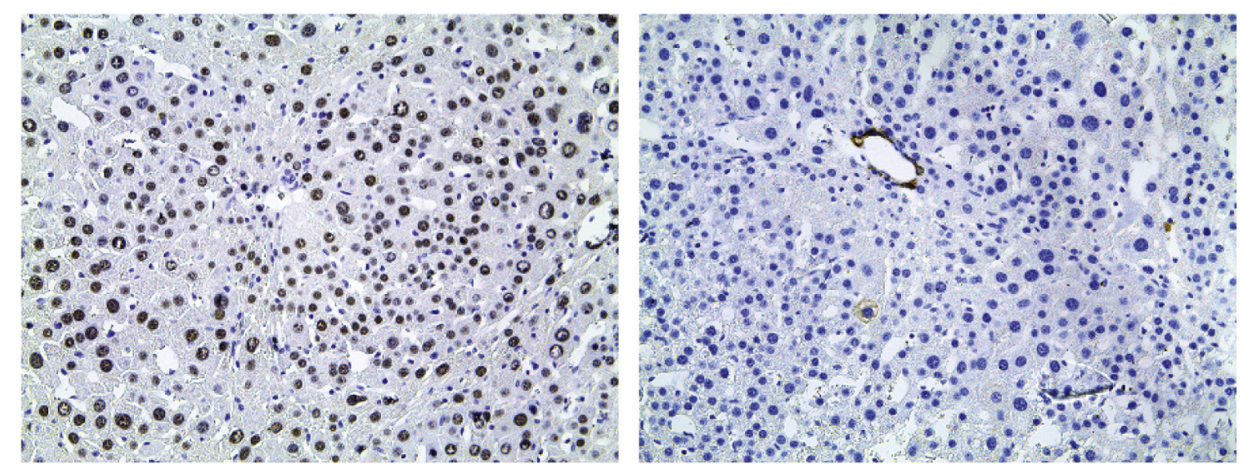

Supplementary Figure 2. All $\beta$-catenin/c-Met-induced foci are positive for the hepatocyte marker HNF4 $\alpha$ but negative for the biliary marker CK19. Original magnifications are shown. 
A

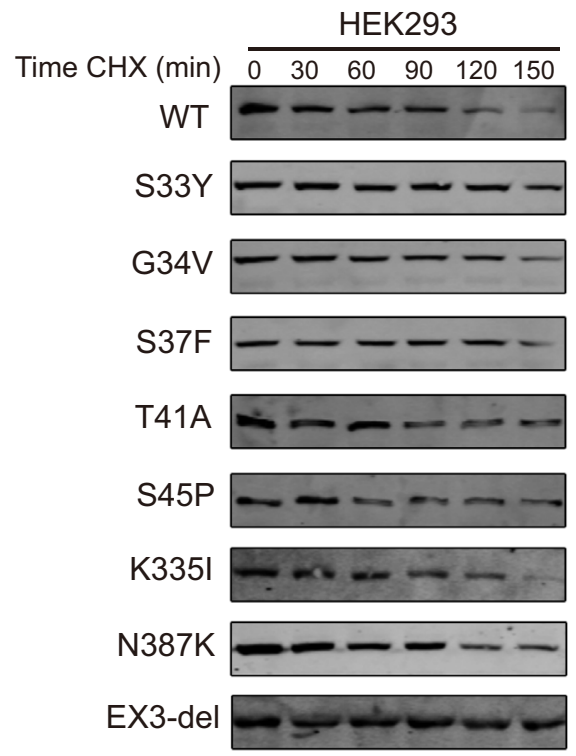

B

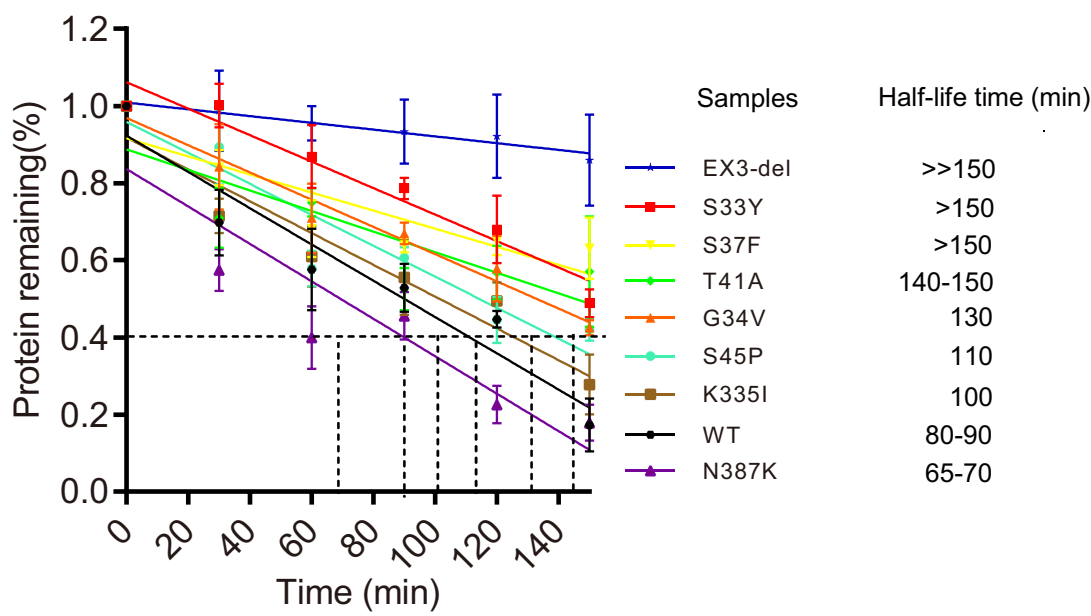

Supplementary Figure 3. Half-life determination of $\beta$-catenin mutants. (A) Indicated FLAG-tagged $\beta$-catenin variants were cotransfected with pEGFP-C1 into HEK293 cells, followed by cycloheximide (CHX) treatment to block new protein synthesis for the indicated times. Next, quantitative fluorescent Western blotting was performed with the highly stable GFP protein for transfection normalization. $(B)$ Values obtained for the 0-hour time point were arbitrarily set to 1 . Normalized values for other time points were plotted. Linear regression was performed to estimate the half-life for each variant, which is depicted on the right. Experiment was performed at least 3 times for each variant producing similar trends in each experiment. EX3-del, exon 3 deletion. 
SNU449 AXIN1 repaired

\section{HEK293}
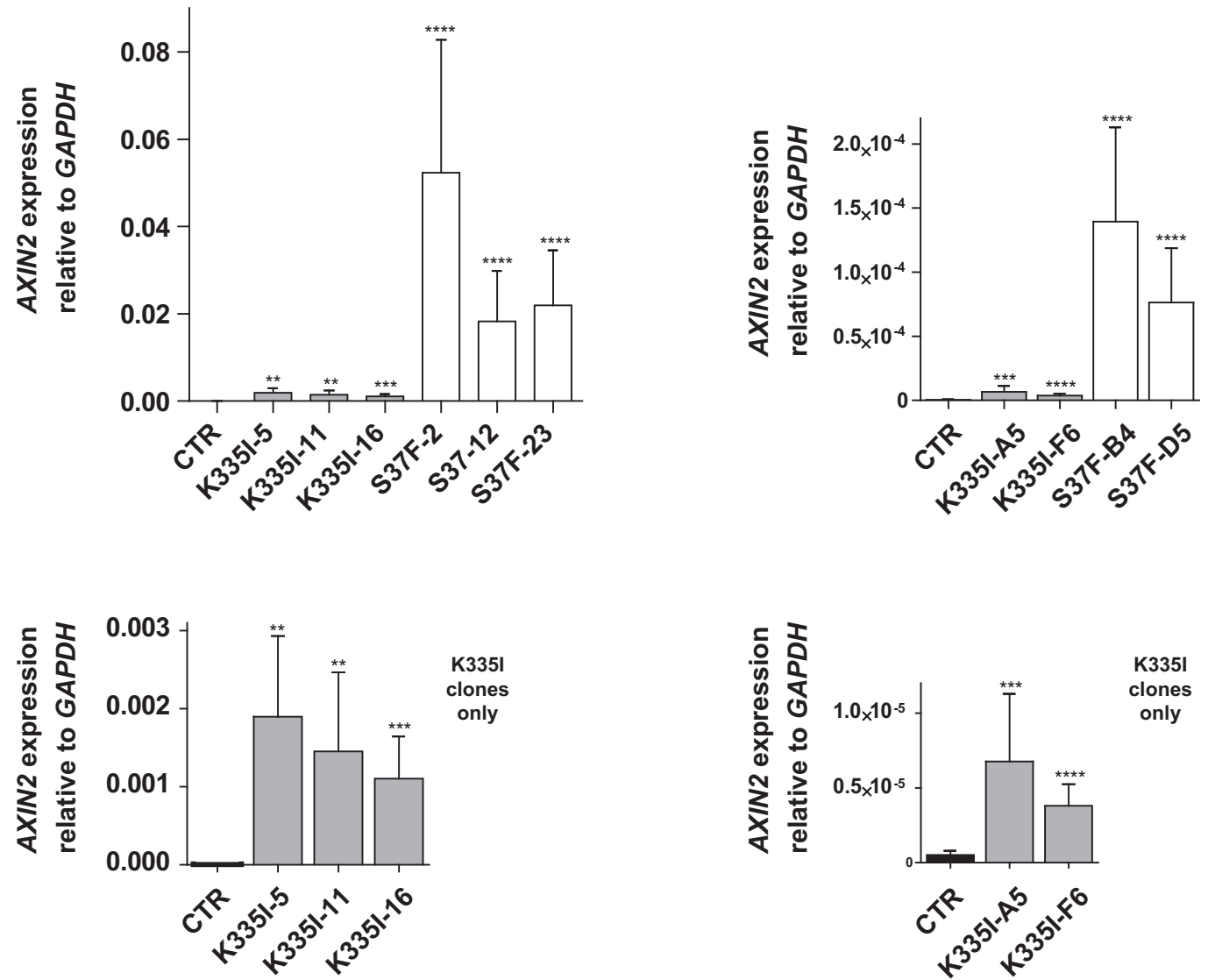

Supplementary Figure 4. AXIN2 RNA expression in $\beta$-catenin S37F and K335I knock-in clones. The top panels show the AXIN2 expression levels determined by qRT-PCR (mean \pm standard deviation, $\mathrm{n}=5$ ) for all analyzed clones. The bottom panels show the results for K335I knock-in clones only. All expression levels are depicted relative to the housekeeping gene GAPDH. Data are presented as mean \pm standard deviation. ${ }^{\star \star} P<.01 ;{ }^{\star \star \star} P<.001 ;{ }^{\star \star \star \star} P<.0001$. CTR, control. 

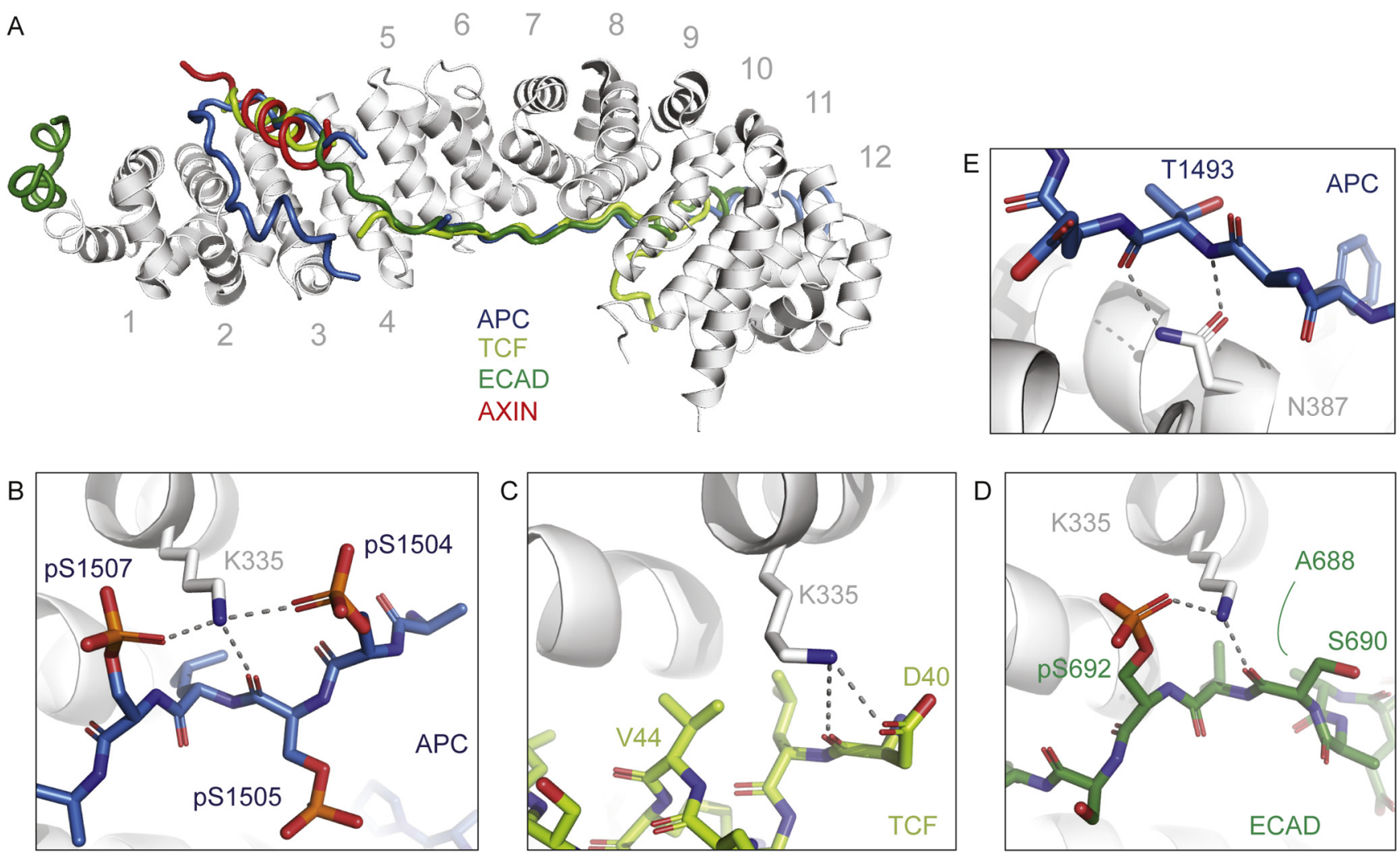

Supplementary Figure 5. K335 and N387 directly interact with APC, TCF/LEF, and E-cadherin. (A) Sketch representation of $\beta$ catenin in gray with its 12 armadillo repeats numbered. Bound APC (blue; 1TH1.pdb), TCF (yellow; 1G3J.pdb), E-cadherin (green; 117W.pdb) and AXIN (red; 1QZ7.pdb) share binding sites in the $\beta$-catenin groove and are shown as tube representation. $(B-E)$ Details of the complexes depicted with $\beta$-catenin as a sketch and bound ligands as stick representation with carbons in the same color code as in panel $A$, oxygen atoms in red, nitrogen atoms in dark blue, phosphorus in orange. ( $B$ ) K335 from $\beta$ catenin forms 2 ionic interactions and 3 hydrogen bonds (dashed lines) with phosphorylated serine residues and backbone atoms of APC. (C) K335 from $\beta$-catenin forms 1 ionic bond and 2 hydrogen bonds (dashed lines) with the sidechain and backbone of D40 in TCF. (D) K335 from $\beta$-catenin forms 1 ionic bond with phosphorylated S692 and a hydrogen bond (dashed line) with the backbone of E-cadherin. (E) N387 from $\beta$-catenin forms 2 hydrogen bonds (dashed lines) with backbone atoms of T1493 in APC. 
A

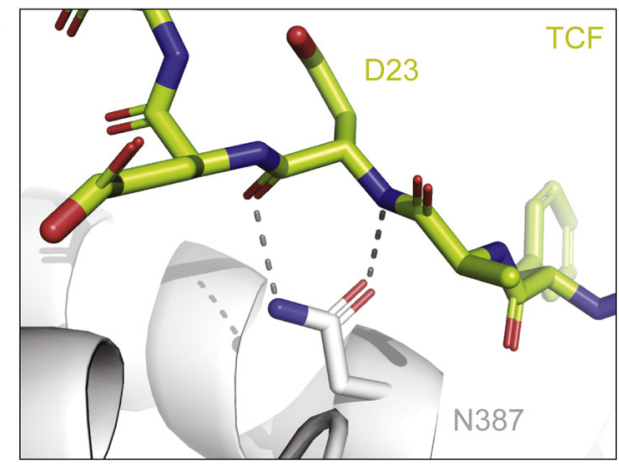

C

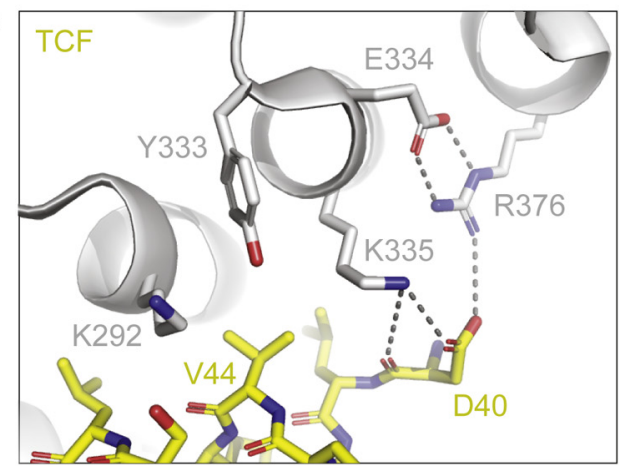

$\mathrm{E}$

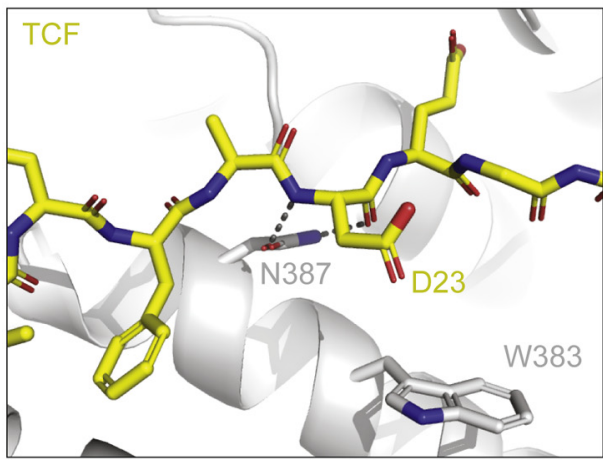

B

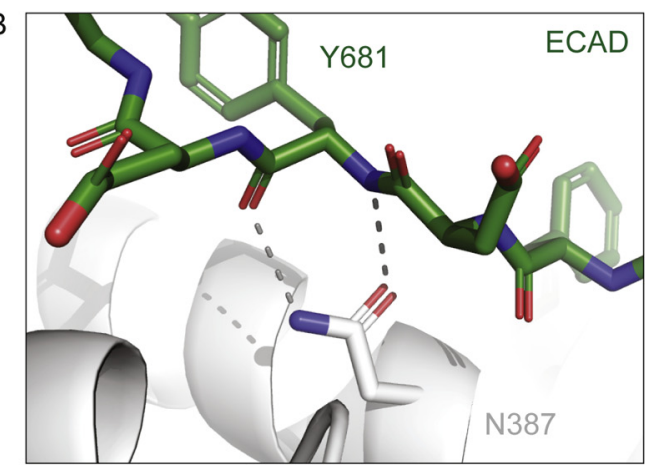

D

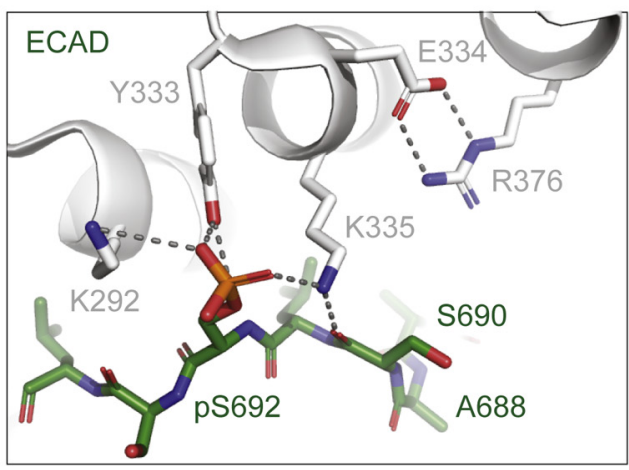

$\mathrm{F}$

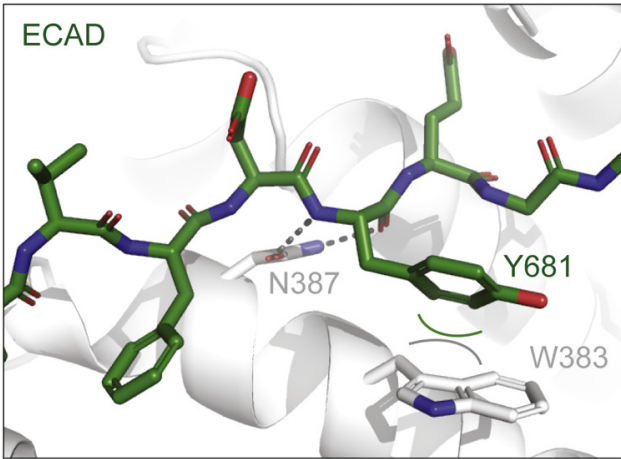

Supplementary Figure 6. Details of TCF and E-cadherin binding to $\beta$-catenin. $(A)$ N387 from $\beta$-catenin forms 2 hydrogen bonds with backbone groups of D23 in TCF. Hydrogen bonds are indicated with dashes. (B) N387 from $\beta$-catenin forms 2 hydrogen bonds with backbone groups of Y681 in E-cadherin. (C) TCF is bound to $\beta$-catenin via a 4-residue ion-pair network (E334-R376-D40-K335). The presence of the hydrophobic V44 sidechain in TCF prevents polar interactions with surrounding residues in $\beta$-catenin. (D) E-cadherin is bound to $\beta$-catenin via a different 4-residue ion-pair network (K292-Y333-pS692-K335). Formation of ionic interactions to connect the 2 isolated networks is impossible because residue 688 in E-cadherin is an alanine rather than a (phosphorylated) serine. $(E)$ The sidechain of D23 in TCF is too short to form energetically favorable interactions with W383 in $\beta$-catenin. $(F)$ The aromatic sidechain of E-cadherin Y681 interacts with the double ring of W383 in $\beta$ catenin via van der Waals interactions (indicated with curved lines). 
Supplementary Table 5. List of Primary Antibodies Used for Immunohistochemistry

\begin{tabular}{|c|c|c|c|c|}
\hline Antibody & Catalog number & Company & Species & Dilution \\
\hline$\beta$-catenin & BD 610153 & BD Biosciences (Franklin Lakes, NJ) & Mouse & $1: 200$ \\
\hline Glutamine synthetase & BD 610518 & BD Biosciences & Mouse & $1: 500$ \\
\hline Myc-tag & Vli01 & Maine Medical Center Research Institute (Portland, ME) & Rabbit & $1: 150$ \\
\hline Ki-67 & MA5-14520 & Thermo Fisher Scientific & Rabbit & $1: 150$ \\
\hline HNF4a & ab181604 & Abcam & Rabbit & $1: 2000$ \\
\hline CK19 & ab133496 & Abcam & Rabbit & $1: 500$ \\
\hline
\end{tabular}

Supplementary Table 6. List of Primer Pairs Used in qRT-PCR Analysis

\begin{tabular}{lll}
\hline Gene name & \multicolumn{1}{c}{ Forward } & Reverse \\
\hline 18s rRNA & CGGCTACCACATCCAAGGAA & GCTGGAATTACCGCGGCT \\
Mouse-Axin2 & GCTCCAGAAGTCACAAAGAGC & AGCTTTGACCTTCAGCATC \\
Mouse-Glul & CAGGCTGCCATACCAACTTCA & TCCTCAATGCACTTCAGACCAT \\
Mouse-Tbx3 & CAGGCAGCCTTCAACTGCTT & GGACACAGATCTTGAGGTTGA \\
Mouse-Afp & TCTGCTGGCACGCAAGAAG & TCGGCAGGTTCTGGAAACTG \\
Mouse-Gpc3 & CAGCCCGGACTCAAATGGG & CAGCCGTGCTGTTAGTTGTA \\
Human-AXIN2 & CAAACTTTCGCCAACCGTGGTTG & GGTGCAAAGACATAGCCAGAACC
\end{tabular}

rRNA, ribosomal RNA.

Supplementary Table 7. List of Antibodies Used for Western Blotting

\begin{tabular}{|c|c|c|c|c|}
\hline Antibody & Catalog number & Company & Species & Dilution \\
\hline FLAG & F3165 & Sigma-Aldrich & Mouse & $1: 1000$ \\
\hline $\mathrm{p} \beta$-catenin (S33/37) & \#2009 & Cell Signaling Technology & Rabbit & $1: 1000$ \\
\hline $\mathrm{p} \beta$-catenin $(\mathrm{S} 33 / 37 / \mathrm{T} 41)$ & \#9561 & Cell Signaling Technology & Rabbit & $1: 1000$ \\
\hline $\mathrm{p} \beta$-catenin (T41/S45) & \#9565 & Cell Signaling Technology & Rabbit & $1: 1000$ \\
\hline $\mathrm{p} \beta$-catenin (S45) & \#9564 & Cell Signaling Technology & Rabbit & $1: 1000$ \\
\hline TCF7L2 & \#05-511 & Merck (Kenilworth, NJ) & Mouse & $1: 1000$ \\
\hline E-cadherin & \#3195 & Cell Signaling Technology & Rabbit & $1: 1000$ \\
\hline Myc-tag & Ab9106 & Abcam & Rabbit & $1: 1000$ \\
\hline GFP & A-11122 & Thermo Fisher Scientific & Rabbit & $1: 1000$ \\
\hline APC & MABC202 (FE9) & Sigma-Aldrich & Mouse & \\
\hline
\end{tabular}

NOTE. Antibody specificities of phospho-specific $\beta$-catenin antibodies as provided by Cell Signaling Technology are as follows: $\mathrm{p}$ - $\beta$ cat Ser33/37 recognizes $\beta$-catenin when phosphorylated at Ser33 or Ser37, or both; $\mathrm{p}$ - $\beta$ cat Ser33/37/Thr41 recognizes any phosphorylation at Ser33/37/Thr41 except for pSer33 alone; $\mathrm{p}-\beta$ cat Thr41/Ser45 recognizes $\mathrm{pThr} 41$ or $\mathrm{pSer} 45$, or both. 
Supplementary Table 8.sgRNA and ssODN Sequences Used to Introduce the S37F and K335I Mutations Into Cell Lines

sgRNA S37F

gCTGGACTCTGGAATCCATTC
GATACTTACGAAAAACTACTG
CCACTCATACAGGACTTGGG
AGGTATCCACATCCTCTT
CCTCAGGATTGCCTTIA
CCACTCAGAGAAGGAGC
TGTGGTAGTGGCACCAAAg
TGAATTCCAGAGTCCAGGT
AAGACTGTTGCTGCCAG
ATAGATACATATACTTACCAGCTT
CTACAATAGCCGGCTTATT
ACTAGAGCAGACAGATAGCA
CCTTCAGCACTCTGCTTGTG
GTCCATAGAATATTTCGTAAGTA
TAGGTCCTCATTATATTTAC

NOTE. The lowercase nucleotides are silent mutations in the ssODNs that will alter the guide RNA recognition sequence to prevent recutting. Underlined and bold $\mathbf{A}$ introduces the S37F and $\mathrm{K} 335 \mathrm{I}$ mutation.

Supplementary Table 9. List of Antibodies Used for Immunoprecipitation

\begin{tabular}{llll}
\hline Antibody & Catalog Number & \multicolumn{1}{c}{ Company } & Species \\
\hline$\beta$-catenin & $\# 8480$ & Cell Signaling Technology & Rabbit \\
APC & $\# 2504$ & Cell Signaling Technology & Rabbit \\
IgG & $\# 2729$ & Cell Signaling Technology & Rabbit \\
APC & AFPN & Homemade $^{4}$ & Rabbit \\
& & & \\
\hline
\end{tabular}

lgG, immunoglobulin $\mathrm{G}$. 